\title{
The Flora of Cunningham Inlet, Somerset Island, Nunavut: History, Analysis, and New Collections of Vascular Plants, Mosses, Lichens, and Algae
}

\author{
Paul C. SoKolofF \\ Centre for Arctic Knowledge and Exploration, Research and Collections, Canadian Museum of Nature, P.O. Box 3443, Sta- \\ tion D, Ottawa Ontario K1P 6P4 Canada; email: psokoloff@mus-nature.ca
}

Sokoloff, Paul C. 2015. The flora of Cunningham Inlet, Somerset Island, Nunavut: history, analysis, and new collections of vascular plants, mosses, lichens, and algae. Canadian Field-Naturalist 129(1): 24-37.

New collections of vascular plants, bryophytes, lichen, and algae are reported for Cunningham Inlet on the north coast of Somerset Island, Nunavut. This list of 48 species of vascular plants, 13 bryophytes, 10 lichens, and five algae includes 136 specimens collected in 2013 and 39 previously unreported specimens from the National Herbarium of Canada at the Canadian Museum of Nature (CAN), Agriculture and Agri-Food Canada's Vascular Plant Herbarium (DAO), and University of Alberta (ALTA). Ten vascular plants from previous collecting in 1958 are re-reported here to give a comprehensive account of the vascular plant flora of the region. Two vascular plants are recorded for the first time for Somerset Island: Smooth Draba (Draba glabella Pursh) and Edlund's Fescue (Festuca edlundiae S. G. Aiken, Consaul \& Lefkovitch).

Key Words: Arctic; Nunavut; Somerset Island; Cunningham Inlet; vascular plants; Festuca edlundiae; Draba glabella

\section{Introduction}

Located on the northern coast of Somerset Island, Nunavut, Cunningham Inlet is a focal point of marine mammal research as thousands of Beluga Whales (Delphinapterus leucas) enter it each summer (Smith and Sjare 1990; Smith and Martin 1994). Because of this remarkable natural phenomenon, Arctic Watch Lodge was established on the inlet as a destination for wilderness tourism. Although this remote area receives numerous visitors and the inlet is close to Canada's high Arctic research hub in Resolute (79 km away), only limited botanical research has previously been carried out at Cunningham Inlet. As a result, relatively few species of vascular plants, mosses, lichens, and algae are reported from the inlet. This might be because of the relative lack of plant diversity on the northern shore of Somerset Island; the Circumpolar Arctic Vegetation Map team classifies this site as cryptogam-herb barren: sparse barren landscapes with little vegetation cover (Walker et al. 2005).

The first significant collecting activity on Somerset Island occurred in 1958, when D. B. O. Savile (Agriculture Canada) collected vascular plants, mosses, and fungi from 12 sites around the perimeter of the island (Savile 1959). Before this, only sporadic collections had been made, many of which are reported by Polunin (1940). Savile visited Cunningham Inlet very briefly on 11 August 1958, and "selective collecting was done, principally of parasitic fungi" (Savile 1959). He collected 11 vascular plants (10 species) and three fungi and deposited them at Agriculture and Agri-Food Canada's, Vascular Plant Herbarium (DAO) and National Mycological Herbarium (DAOM). These collections were reported in Savile (1959), and a subset was mapped for
Flora of the Canadian Arctic Archipelago (Aiken et al. 2007). These specimens may have also been mapped by Porsild and Cody (1980), but they are difficult to distinguish among the individual dots that cover nearly the entirety of Somerset Island on their location maps.

Before Savile's work, only one known specimen had been collected from Cunningham Inlet: a single sheet of Arctic False Wallflower (Parrya arctica R. Brown) collected by B. Shindman during "Operation Magnetic" in 1949.

Over a decade passed until the next collector, L. C. Bliss of the University of Alberta, visited Cunningham Inlet to study the plant communities in polar desert habitats (Bliss et al. 1984). His 23 previously unreported specimens (19 vascular plant species) are deposited at the University of Alberta herbarium (ALTA).

Steven V. Zoltai and V. Woo conducted extensive soil and vegetation reconnaissance on Somerset Island for a proposed gas pipeline through the Canadian Arctic in 1977 (Woo and Zoltai 1977). While they used field identifications from plots and transects to characterize the vegetation of habitats sampled, voucher specimens were taken and sent to Agriculture Canada (DAO), including three species of Poaceae from the vicinity of Cunningham Inlet.

More recently, on 13 July 2004, L. Consaul and A. Archambault of the Canadian Museum of Nature made a brief stop at Cunningham Inlet and collected 11 previously unreported specimens for the National Herbarium of Canada (CAN), focusing primarily on False Wallflowers (Parrya R. Brown) and Alkaligrasses (Puccinellia Parlatore). In total, they collected four vascular plant species. 
In July 2013, I embarked on a 6-day plant collecting trip to Cunningham Inlet as a scientist-in-residence for Arctic Watch Lodge's 2013 Steve Amarualik Youth Leadership Expedition, a program designed to bring out leadership potential and teach outdoor skills to youth from the south and the north. The collections from this trip are described here alongside the collections made on the four previously mentioned trips. This provides a comprehensive overview of the vascular plant flora on the inlet, which consists of 48 species and 31 genera in 11 plant families. I include two vascular plant species previously not known to occur on Somerset Island. In addition, I report here the first substantial inventory of cryptogams and marine algae from Cunningham Inlet.

\section{Study Area}

From 6 to 12 July 2013, I collected vascular plants, lichens, mosses, algae, and fungi in the vicinity of Cunningham Inlet $\left(74^{\circ} 04^{\prime} \mathrm{N}, 93^{\circ} 48^{\prime} \mathrm{W}\right.$; Figure 1$)$.

\section{Methods}

I collected specimens of all vascular plant and marine algae species encountered and opportunistically collected mosses, lichens, and fungi (Figure 2). Two stu- dents, Zachary Halem (New York, New York, USA) and Alicia Manik (Resolute, Nunavut, Canada), provided extensive assistance with the collection and found additional specimens; thus they are named as collectors where appropriate. In all, 136 numbers were collected: 93 vascular plants (11 families, 31 genera, and 48 species), 12 bryophytes (eight families, 13 genera, and 13 species), 16 marine algae and terrestrial cyanobacteria (five families, five genera, and five species), 12 lichens (seven families, 10 genera, and 10 species), and two fungi. All specimens have been deposited in the relevant collection (CAN for vascular plants, CANM for bryophytes, CANL for lichens, and CANA for algae) in the National Herbarium of Canada at the Canadian Museum of Nature, except the fungal collections, which were deposited at DAOM. Herbaria with duplicate specimens are indicated in the species accounts by their herbarium acronym. Twelve unidentified vascular plant, lichen, algae, and fungal specimens collected during the trip are not treated in this paper: Sokoloff 149, 159, 162, 177, 179, 194, 195, 196, 208, 222, 229, and 241 .

I also examined and verified the vascular plant collections made by Savile (11 numbers), Bliss (24 num-

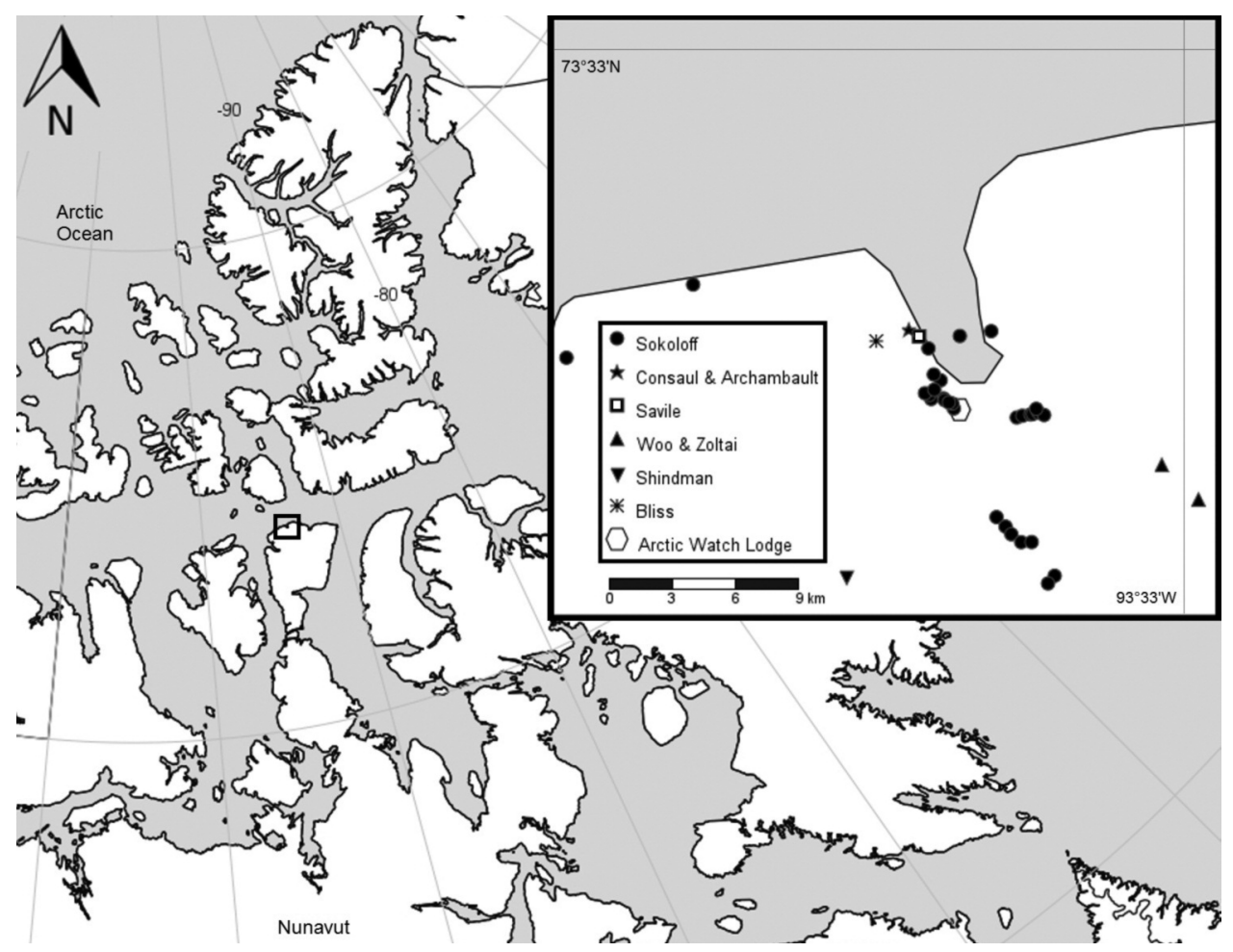

FIGURE 1: Locations of vascular plant, algae, fungi, and bryophyte collections made in the Cunningham Inlet area, Somerset Island, Nunavut. Locations of earlier collections (Sokoloff, Consaul \& Archambault, Savile, Woo \& Zoltai, Shindman, and Bliss) and Arctic Watch Lodge are denoted by symbols (right inset). There is a peninsula on the northeast corner of Cunningham Inlet not shown due to map resolution. 

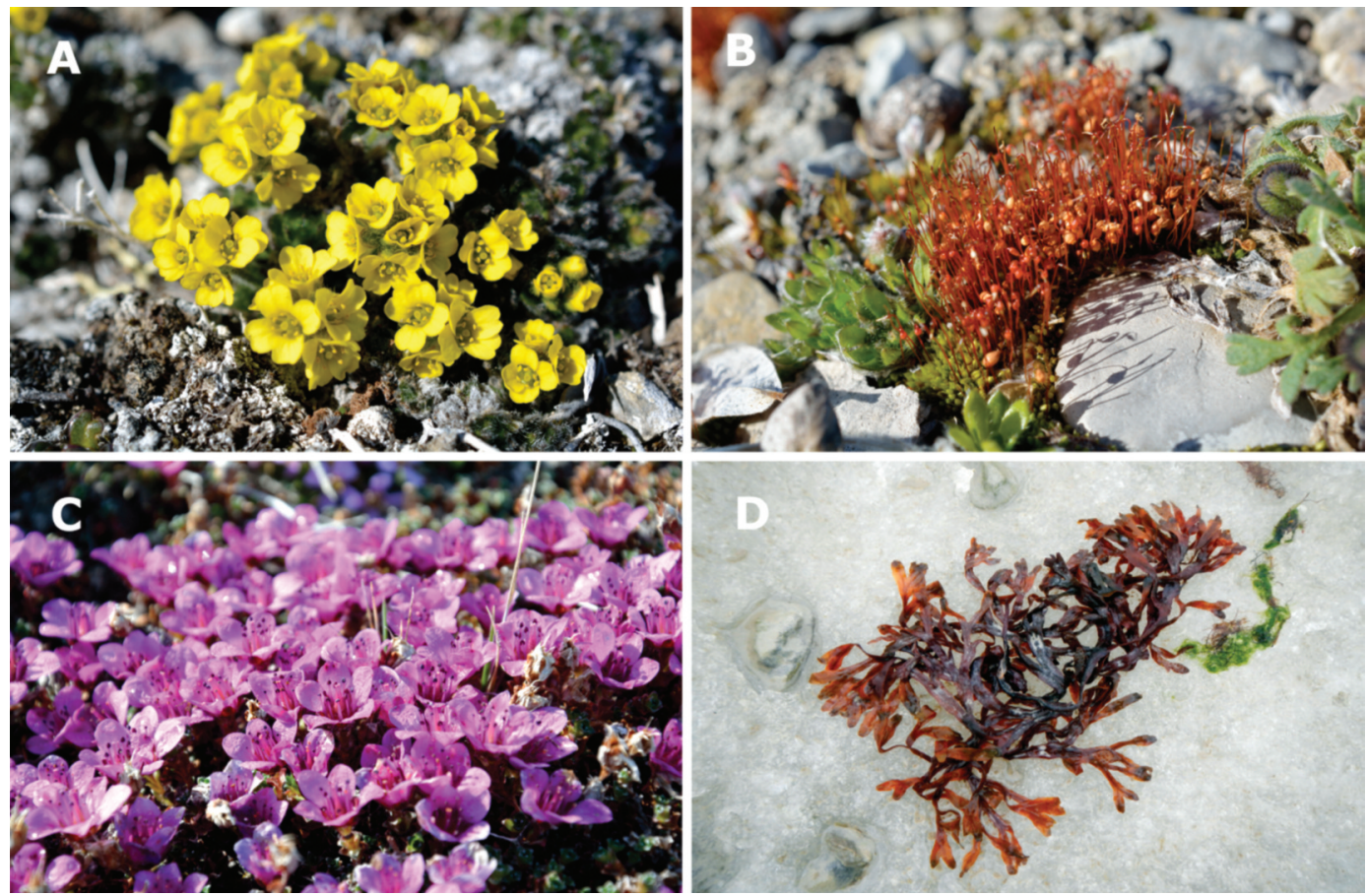

FIGURE 2: Diverse specimens from the Cunningham Inlet area, Somerset Island, Nunavut: A) Flat-top Draba (Draba corymbosa R. Brown ex de Candolle), Sokoloff 245; B) Ptychostomum wrightii, Sokoloff 187, C) Purple Mountain Saxifrage (Saxifraga oppositifolia L.), Sokoloff 181, D) Fucus distichus, Sokoloff 140. Photos by P. Sokoloff.

bers), Woo and Zoltai (3 numbers), Shindman (1 number), and Consaul and Archambault (11 numbers) and report all except Savile's for the first time. Some material was examined and identified by specialists, who are listed in the acknowledgements.

The species accounts are organized alphabetically by family, genus, and species within each major group collected: algae, bryophytes, lichens, and vascular plants. Common names for vascular plant species in English and French are taken from Vascan (Brouillet et al. 2010*). Inuktitut names are taken from the Common Plants of Nunavut (Mallory and Aiken 2004). Collections were plotted using SimpleMappr (opensource software, David P. Shorthouse, http://www.sim plemappr.net) (Figure 1). Species distributions are taken from the Annotated Checklist of the Panarctic Flora (Elven et al. 2011*).

\section{Results}

Diversity in the algae, bryophyte, and lichen collections was relatively low, and the opportunistic sampling strategy makes it difficult to compare our collections with known checklists from the area. Five species of algae were documented, in five genera representing five families (not including unidentified samples). For bryophytes, 14 genera and species from eight families were collected, and 10 species and genera from seven families of lichens were documented.
For vascular plants, 48 species in 31 genera and 11 families were documented for Cunningham Inlet. This accounts for nearly half of the 98 species recorded for Somerset Island by Savile (1959) and the 75 species recorded by Woo and Zoltai (1959) and represents 40\% of the 119 species documented for the Island in Flora of the Canadian Arctic Archipelago (Aiken et al. 2007, data obtained from species maps). At Cunningham Inlet, Poaceae, Brassicaceae, and Caryophyllaceae were the most species-rich families, accounting for $25 \%$, $23 \%$, and $15 \%$ of the species collected. The majority of vascular plants documented (65\%) possess a circumpolar distribution pattern; others are amphi-Beringian and North American species.

\section{Description of specimens}

AlgAe

\section{Alariacaeae}

Alaria esculenta (L.) Greville - NunAvUT: Somerset Island, in sea ice in centre of Cunningham Inlet, $3 \mathrm{~km}$ north of Arctic Watch Lodge, thick sea ice with algae embedded, $74^{\circ} 5^{\prime} 54^{\prime \prime} \mathrm{N}, 93^{\circ} 47^{\prime} 15^{\prime \prime} \mathrm{W}, 0 \mathrm{~m}, 10$ July 2013, P. Sokoloff 192 (CANA 93578).

\section{Costariaceae}

Agarum cribrosum Bory de Saint-Vincent - NunAVUT: Somerset Island, on tidal flats on west edge of Cunningham Inlet, $1.5 \mathrm{~km}$ north of Arctic Watch 
Lodge, in rocky tidal zone, mostly bare stones, with Fucus distichus, 744'48.7"N, 9349'27.7"W, 53 m, 8 July 2013, P. Sokoloff 139 (CANA 93579); sea ice in centre of Cunningham Inlet, $3 \mathrm{~km}$ north of Arctic Watch Lodge, thick sea ice with algae embedded, $74^{\circ} 5^{\prime} 54^{\prime \prime} \mathrm{N}$, 934' 15"W, 0 m, 10 July 2013, P. Sokoloff 193 (CANA 93580).

\section{Fucaceae}

Fucus distichus L. - NunAVUT: Somerset Island, tidal flats on west edge of Cunningham Inlet, $1.5 \mathrm{~km}$ north of Arctic Watch Lodge, rocky tidal zone, mostly bare stones, $74^{\circ} 4^{\prime} 48.7^{\prime \prime} \mathrm{N}, 93^{\circ} 49^{\prime} 27.7^{\prime \prime} \mathrm{W}, 53 \mathrm{~m}, 8$ July 2013, P. Sokoloff 140 (CANA 93581); tidal flats on west edge of Cunningham Inlet, $1.5 \mathrm{~km}$ north of Arctic Watch Lodge, rocky tidal zone, mostly bare stones, 74²'48.7"N, 9349'27.7"W, 53 m, 8 July 2013, $P$. Sokoloff 141 (CANA 93582); tidal flats on west edge of Cunningham Inlet, $1.5 \mathrm{~km}$ north of Arctic Watch Lodge, rocky tidal zone, mostly bare stones, $74^{\circ} 4^{\prime} 48.7^{\prime \prime N}$, 9349'27.7"W, 53 m, 8 July 2013, P. Sokoloff 142 (CANA 93583); tidal flats on west edge of Cunningham Inlet, $1.5 \mathrm{~km}$ north of Arctic Watch Lodge, rocky tidal zone, mostly bare stones, $74^{\circ} 4^{\prime} 48.7^{\prime \prime} \mathrm{N}, 93^{\circ} 49^{\prime} 27.7^{\prime \prime} \mathrm{W}$, 53 m, 8 July 2013, P. Sokoloff 143 a (CANA 93584); sea ice in centre of Cunningham Inlet, $3 \mathrm{~km}$ north of Arctic Watch Lodge, thick sea ice with algae embedded, $74^{\circ} 5^{\prime} 54^{\prime \prime} \mathrm{N}, 93^{\circ} 47^{\prime} 15^{\prime \prime} \mathrm{W}, 0 \mathrm{~m}, 10$ July 2013, $P$. Sokoloff 191 (CANA 93585).

\section{Laminariaceae}

Saccharina latissima (L.) C.E. Lane, C. Mayes, Druehl \& G.W. Saunders - NunAVUT: Somerset Island, sea ice in centre of Cunningham Inlet, $3 \mathrm{~km}$ north of Arctic Watch Lodge, thick sea ice with algae embedded, $74^{\circ} 5^{\prime} 54^{\prime \prime} \mathrm{N}, 93^{\circ} 47^{\prime} 15^{\prime \prime} \mathrm{W}, 0 \mathrm{~m}, 10$ July $2013, P$. Sokoloff 190 (CANA 93586).

\section{Nostocaceae}

Nostoc commune Vaucher ex Bornet \& Flahault NUNAVUT: Somerset Island, north-facing ridge west of Arctic Watch Lodge, Eriophorum-Calamagrostis meadow, 744'27.7"N, 9350'39.4"W, 118 m, 9 July 2013, P. Sokoloff 175 (CANA 93588); north-facing ridge west of Arctic Watch Lodge, Eriophorum-Calamagrostis meadow, $74^{\circ} 4^{\prime} 27.7^{\prime \prime} \mathrm{N}, 93^{\circ} 50^{\prime} 39.4^{\prime \prime} \mathrm{W}, 118 \mathrm{~m}, 9$ July 2013, P. Sokoloff 176 (CANA 93587).

\section{BRYOPHYTES}

\section{Amblystegiaceae}

Campylium stellatum (Hedwig) Christian Erasmus Otterstrøm Jensen - NunAVUT: Somerset Island, 2 km south of point at Cape Anne, muddy wet ground in marshy field alongside river, with Saxifraga oppositifolia, Salix arctica, Dryas integrifolia, $74^{\circ} 6^{\prime} 23.3^{\prime \prime} \mathrm{N}$, 94²3'44.25"W, 26 m, 10 July 2013, P. Sokoloff, Z. Halem 239 (associated species in same packet as Ditrichum flexicaule) (CANM 332657).

Scorpidium revolvens (O.P. Swartz ex Anonymo) W.V. Rubers in A. Touw \& W.V. Rubers - NunAVUT: Somerset Island, wet snowmelt valley at base of large unnamed mountain northwest of Arctic Watch Lodge, wet mossy tundra, with Eriophorum angustifolium, $74^{\circ} 4^{\prime} 31.3^{\prime \prime N}, 93^{\circ} 51^{\prime} 1.7^{\prime \prime} \mathrm{W}, 122 \mathrm{~m}, 9$ July 2013, . Sokoloff 184 (CANM 332651).

Drepanocladus sordidus (Müller Hal.) Hedenäs NunAVUT: Somerset Island, south end of Sunday Lake, $7 \mathrm{~km}$ south of Arctic Watch Lodge, wet Eriophorum meadow, $74^{\circ} 0^{\prime} 24.9^{\prime \prime} \mathrm{N}, 93^{\circ} 43^{\prime} 40.9^{\prime \prime} \mathrm{W}, 26 \mathrm{~m}, 11$ July 2013, P. Sokoloff 220 (CANM 332655); slope above alluvial plain of Cunningham River, $4 \mathrm{~km}$ east of Arctic Watch Lodge, mossy bank in wet sedge mead-

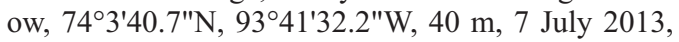
P. Sokoloff 124 (associated species in same packet as Brachythecium cirrosum) (CANM 332646).

Hygrohypnum luridum (Hedwig) Jennings - NunAVUT: Somerset Island, Flat Rock Falls, east coast of Cunningham Inlet, $4 \mathrm{~km}$ northeast of Arctic Watch Lodge, wet seepy rocks at edge of falls, $74^{\circ} 5^{\prime} 56.1^{\prime \prime} \mathrm{N}$, 934' 18.5"W, 55 m, 10 July 2013, P. Sokoloff 204 (CANM 332653).

\section{Brachytheciaceae}

Brachythecium cirrosum (Schwägrichen) Schimper - NunAVUT: Somerset Island, slope above alluvial plain of Cunningham River, $4 \mathrm{~km}$ east of Arctic Watch Lodge, mossy bank in wet sedge meadow, $74^{\circ} 3^{\prime} 40.7^{\prime \prime} \mathrm{N}$, $93^{\circ} 41^{\prime} 32.2^{\prime \prime W}, 40 \mathrm{~m}, 7$ July 2013, P. Sokoloff 124 (CANM 332646).

\section{Bryaceae}

Ptychostomum wrightii (Sullivant) J.R. Spence NunAVUT: Somerset Island, garden spot below sewage lagoon at Arctic Watch Lodge, lush green patch in rocky scree, with Salix arctica, Saxifraga oppositifolia, Papaver sp., Parrya arctica, $74^{\circ} 4^{\prime} 13.1^{\prime \prime} \mathrm{N}, 93^{\circ} 48^{\prime} 55.8^{\prime \prime} \mathrm{W}$, 16 m, 9 July 2013, P. Sokoloff 187 (CANM 332652); south end of Sunday Lake, $7 \mathrm{~km}$ south of Arctic Watch Lodge, wet Eriophorum meadow, $74^{\circ} 0^{\prime} 24.9^{\prime \prime} \mathrm{N}$, 934' 40.9"W, 26 m, 11 July 2013, P. Sokoloff 221 (CANM 332656).

\section{Ditrichaceae}

Distichium capillaceum (Hedwig) Bruch \& Schimper - NunAVUT: Somerset Island, confluence of Cunningham River and stream immediately south of Arctic Watch Lodge at Cunningham River crossing, rocky talus on south-facing slope, $74^{\circ} 4^{\prime} 2.9^{\prime \prime} \mathrm{N}, 93^{\circ} 48^{\prime} 31.7^{\prime \prime} \mathrm{W}, 58 \mathrm{~m}$, 8 July 2013, P. Sokoloff 147 (associated species in same packet as Ditrichum flexicaule) (CANM 332649).

Ditrichum flexicaule (Schwägrichen) Hampe NunAVUT: Somerset Island, sloping west wall at Gull Canyon over dry creek bed, $4 \mathrm{~km}$ east of Arctic Watch Lodge, wet mossy slope, with Saxifraga oppositifolia, Cerastium arcticum, Draba glabella, $74^{\circ} 3^{\prime} 37.5^{\prime \prime} \mathrm{N}$, 9340'17.7"W, 40 m, 7 July 2013, P. Sokoloff 132 (CANM 332647); confluence of Cunningham River and stream immediately south of Arctic Watch Lodge at Cunningham River crossing, rocky talus on southfacing slope, with Cerastium alpinum, $74^{\circ} 4^{\prime} 2.9^{\prime \prime} \mathrm{N}$, 93²4'31.7"W, 58 m, 8 July 2013, P. Sokoloff 147 
(CANM 332649); $2 \mathrm{~km}$ south of point at Cape Anne, muddy wet ground in marshy field alongside river, with Saxifraga oppositifolia, Salix arctica, Dryas integrifolia, $74^{\circ} 6^{\prime} 23.3^{\prime \prime} \mathrm{N}, 94^{\circ} 23^{\prime} 44.25^{\prime \prime} \mathrm{W}, 26 \mathrm{~m}, 10$ July 2013 , P. Sokoloff, Z. Halem 239 (associated species in same packet as Orthothecium chryseum) (CANM 332657).

\section{Grimmiaceae}

Schistidium rivulare (Bridel) Podpera - NunavuT: Somerset Island, Flat Rock Falls, east coast of Cunningham Inlet, $4 \mathrm{~km}$ northeast of Arctic Watch Lodge, dry rocks at edge of falls, $74^{\circ} 5^{\prime} 56.1^{\prime \prime} \mathrm{N}, 93^{\circ} 44^{\prime} 18.5^{\prime \prime} \mathrm{W}$, 55 m, 10 July 2013, P. Sokoloff 205 (CANM 332654).

\section{Hypnaceae}

Orthothecium chryseum (Schwägrichen in Schultes) Schimper in Bruch \& Schimper - NunAvUT: Somerset Island, $2 \mathrm{~km}$ south of point at Cape Anne, muddy wet ground in marshy field alongside river, with Saxifraga oppositifolia, Salix arctica, Dryas integrifolia, $74^{\circ} 6^{\prime} 23.3^{\prime \prime} \mathrm{N}, 94^{\circ} 23^{\prime} 44.25^{\prime \prime} \mathrm{W}, 26 \mathrm{~m}, 10$ July $2013, P$. Sokoloff, Z. Halem 239 (CANM 332657); slope above alluvial plain of Cunningham River, $4 \mathrm{~km}$ east of Arctic Watch Lodge, mossy bank in wet sedge meadow, 743' $40.7^{\prime \prime} \mathrm{N}, 93^{\circ} 41^{\prime} 32.2^{\prime \prime} \mathrm{W}, 40 \mathrm{~m}, 7$ July 2013, $P$. Sokoloff 124 (associated species in same packet as Brachythecium cirrosum) (CANM 332646).

\section{Mniaceae}

Cinclidium cf. arcticum (Bruch \& Schimper) Schimper-NunAVUT: Somerset Island, $2 \mathrm{~km}$ south of point at Cape Anne, muddy wet ground in marshy field alongside river, with Saxifraga oppositifolia, Salix arctica, Dryas integrifolia, $74^{\circ} 6^{\prime} 23.3^{\prime \prime} \mathrm{N}, 94^{\circ} 23^{\prime} 44.25^{\prime \prime} \mathrm{W}, 26 \mathrm{~m}$, 10 July 2013, P. Sokoloff, Z. Halem 239 (associated species in same packet as Orthothecium chryseum) (CANM 332657).

Mnium blyttii Bruch \& Schimper - Nunavut: Somerset Island, eastern edge of Gull Canyon, $4 \mathrm{~km}$ east of Arctic Watch Lodge, extremely deep moss directly above snow line, $74^{\circ} 3^{\prime} 48.4^{\prime \prime} \mathrm{N}, 93^{\circ} 40^{\prime} 57.2^{\prime \prime} \mathrm{W}, 40 \mathrm{~m}$, 7 July 2013, P. Sokoloff 137 (CANM 332648).

\section{Scorpidiaceae}

Sanionia uncinata (Hedwig) Loeske - NunAvut: Somerset Island, western edge of Gull Canyon, $4 \mathrm{~km}$ east of Arctic Watch Lodge, extremely deep moss directly above snow line, $74^{\circ} 3^{\prime} 48.4^{\prime \prime} \mathrm{N}, 93^{\circ} 40^{\prime} 57.2^{\prime \prime} \mathrm{W}$, 40 m, 7 July 2013, P. Sokoloff 137 (associated species in same packet as Mnium blyttii) (CANM 332648).

\section{LICHENS}

\section{Icmadophilaceae}

Thamnolia subuliformis (Ehrhart) W.L. Culberson - NunAvut: Somerset Island, mossy wet area outside whale biologist's cabin, $1.5 \mathrm{~km}$ north of Arctic Watch Lodge, wet mossy rocks, $74^{\circ} 4^{\prime} 58.8^{\prime \prime} \mathrm{N}, 93^{\circ} 50^{\prime} 2 " \mathrm{~W}$, 94 m, 8 July 2013, P. Sokoloff 157 (CANL 125971).

\section{Lecanoraceae}

Lecidella patavina (A. Massalongo) Knoph \& Leuckert - NUNAVUT: Somerset Island, rocky beach on west coast of Cunningham Inlet, directly across from Flat Rock Falls $4 \mathrm{~km}$ north of Arctic Watch Lodge, barren rocks, $74^{\circ} 5^{\prime} 40^{\prime \prime} \mathrm{N}, 93^{\circ} 50^{\prime} 16^{\prime \prime} \mathrm{W}, 5 \mathrm{~m}, 10$ July 2013, P. Sokoloff 211 (CANL 125978).

\section{Megasporaceae}

Aspicilia candida (Anzi) Hue - NunAVut: Somerset Island, Flat Rock Falls, east coast of Cunningham Inlet, $4 \mathrm{~km}$ northeast of Arctic Watch Lodge, dry gravel scree above falls, $74^{\circ} 5^{\prime} 56.1^{\prime \prime} \mathrm{N}, 93^{\circ} 44^{\prime} 18.5^{\prime \prime} \mathrm{W}, 55 \mathrm{~m}, 10$ July 2013, P. Sokoloff, R. Weber 206 (CANL 125980).

Megaspora verrucosa (Acharius) Hafellner \& V. Wirth - NunAVuT: Somerset Island, mossy wet area outside whale biologist's cabin, $1.5 \mathrm{~km}$ north of Arctic Watch Lodge, wet mossy rocks, with Saxifraga cernua, Luzula confusa, Draba sp., Salix arctica, $74^{\circ} 4^{\prime} 58.8^{\prime \prime} \mathrm{N}$, 9350'2"W, 94 m, 8 July 2013, P. Sokoloff 160 (CANL 125974); mossy wet area outside whale biologist's cabin, $1.5 \mathrm{~km}$ north of Arctic Watch Lodge, wet mossy rocks, with Saxifraga cernua, Luzula confusa, Draba sp., Salix arctica, $74^{\circ} 4^{\prime} 58.8^{\prime \prime N}, 93^{\circ} 50^{\prime} 2^{\prime \prime W}, 94$ m, 8 July 2013, P. Sokoloff 163 (CANL 125976).

\section{Parmeliaceae}

Allocetraria madreporiformis (Withering) Kärnefelt \& A. Thell - NunAvuT: Somerset Island, mossy wet area outside whale biologist's cabin, $1.5 \mathrm{~km}$ north of Arctic Watch Lodge, wet mossy rocks, with Saxifraga cernua, Luzula confusa, Draba sp., Salix arctica, $74^{\circ} 4^{\prime} 58.8^{\prime \prime} \mathrm{N}, 93^{\circ} 50^{\prime} 2^{\prime \prime} \mathrm{W}, 94 \mathrm{~m}, 8$ July 2013, $P$. Sokoloff 158 (CANL 125972).

Evernia divaricata (L.) Acharius - NunAVUT: Somerset Island, windswept dry rocks on ridge off northwest coast of Cunningham Inlet, dry rocky talus, with Saxifraga oppositifolia, $74^{\circ} 4^{\prime} 35.3^{\prime \prime} \mathrm{N}, 93^{\circ} 50^{\prime} 7.9^{\prime \prime} \mathrm{W}$, 98 m, 9 July 2013, P. Sokoloff 186 (CANL 125977).

Vulpicida tilesii (Acharius) J.-E. Mattsson \& M.J. Lai - NunAVuT: Somerset Island, west ridge overlooking Gull Canyon, 4 km east of Arctic Watch Lodge, dry rocky scree, with Festuca sp., Saxifraga oppositifolia, 74³'42.4"N, 9304'47.8"W, 50 m, 7 July 2013, $P$. Sokoloff 129 (CANL 125969).

\section{Peltigeraceae}

Peltigera ponojensis Gyelnik - NunAvUT: Somerset Island, south end of Sunday Lake, $6 \mathrm{~km}$ south of Arctic Watch Lodge, mossy knoll in wet tundra, $74^{\circ} 1^{\prime} 7.8^{\prime \prime} \mathrm{N}$, 934ㄴ'41.5"W, 66 m, 11 July 2013, P. Sokoloff 214 (CANL 125979).

\section{Physciaceae}

Physcia dubia (Hoffmann) Lettau - NunAvuT: Somerset Island, west ridge overlooking Gull Canyon, 4 $\mathrm{km}$ east of Arctic Watch Lodge, dry rocky scree, with Festuca sp., Saxifraga oppositifolia, 74³'42.4"N, $93^{\circ} 40^{\prime} 47.8^{\prime W}, 50$ m, 7 July 2013, P. Sokoloff 130 (CANL 125970).

\section{Teloschistaceae}

Xanthoria elegans (Link) Th. Fries - NunAVUT: Somerset Island, mossy wet area outside whale biol- 
ogist's cabin, $1.5 \mathrm{~km}$ north of Arctic Watch Lodge, wet mossy rocks, with Saxifraga cernua, Luzula confusa, Draba sp., Salix arctica, 744'58.8"N, 9350'2"W, 94 m, 8 July 2013, P. Sokoloff 161 (CANL 125975).

\section{Vascular Plants}

\section{Brassicaceae}

Braya glabella Richardson ssp. purpurascens (R. Brown) W.J. Cody (Purple Braya, braya purpurine, Airaujuit) [circumpolar-cordilleran] - NunAVUT: Somerset Island, on a mild slope, with Dryas integrifolia, Salix, 746'00"N, 9351'00"W, 63 m, July 13, 2004, $A$. Archambault \& L. Consaul aa53 (CAN 603400); west ridge overlooking Gull Canyon, $4 \mathrm{~km}$ east of Arctic Watch Lodge, tundra in dry mud, with Dryas integrifolia, $74^{\circ} 3^{\prime} 41.1^{\prime \prime} \mathrm{N}, 93^{\circ} 41^{\prime} 18.8^{\prime \prime} \mathrm{W}, 50 \mathrm{~m}, 7$ July 2013, $P$. Sokoloff 125 (CAN 603292).

Cardamine bellidifolia L. (Alpine Bittercress, cardamine à feuilles de pâquerette) [circumpolar-alpine] - NunAvut: Somerset Island, Flat Rock Falls, east coast of Cunningham Inlet, $4 \mathrm{~km}$ northeast of Arctic Watch Lodge, wet snow-patch community at foot of packed snowbank, near foot of falls, $74^{\circ} 5^{\prime} 56.1^{\prime \prime} \mathrm{N}$, 934' 18.5"W, 55 m, 10 July 2013, P. Sokoloff 197 (CAN 603293); south end of Sunday Lake, north of Cunningham River, $7 \mathrm{~km}$ south of Arctic Watch Lodge, mud flats, $74^{\circ} 0^{\prime} 23.3^{\prime \prime} \mathrm{N}, 93^{\circ} 42^{\prime} 43.5^{\prime \prime} \mathrm{W}, 41 \mathrm{~m}, 11$ July 2013, P. Sokoloff 230 (CAN 603294).

Cochlearia groenlandica L. (Greenland Scurvygrass, cranson du Groenland, Tipitsiariktut nunarait) [circumpolar] - NUNAVUT: Somerset Island, south end of Sunday Lake, north of Cunningham River, $7 \mathrm{~km}$ south of Arctic Watch Lodge, mud flats, $74^{\circ} 0 ' 23.3^{\prime \prime N}$, $93^{\circ} 42^{\prime} 43.5^{\prime \prime W}, 41 \mathrm{~m}, 11$ July 2013, P. Sokoloff 233 (CAN 603295).

Draba corymbosa R. Brown ex de Candolle (Flattop Draba, drave en corymbe) [circumpolar] - Cunningham Inlet, $8 \mathrm{~km}$ from inlet, uplands, polar desert, Somerset plateau, $74^{\circ} 06^{\prime} \mathrm{N}, 93^{\circ} 55^{\prime} \mathrm{W}, 250 \mathrm{~m}, 20$ July 1976, L.C. Bliss s.n. (ALTA 56724); Cunningham Inlet, $8 \mathrm{~km}$ from inlet, uplands, polar desert, $74^{\circ} 06^{\prime} \mathrm{N}$, $93^{\circ} 55^{\prime} \mathrm{W}, 225 \mathrm{~m}, 20$ July 1976, L.C. Bliss s.n. (ALTA 56723); dry stone ridge immediately west of Arctic Watch Lodge, in gravel scree, dry gravel, with Saxifraga oppositifolia, Salix arctica, Papaver sp., $74^{\circ} 4^{\prime} 20.2^{\prime \prime N}, 93^{\circ} 49^{\prime} 22.6^{\prime \prime} \mathrm{W}, 20 \mathrm{~m}, 6$ July 2013 , P. Sokoloff 112 (CAN 603296); western cliff wall of Gull Canyon, below gull nesting area, wet rocks directly under water seeps and waterfall, dense lush vegetation, with Saxifraga cespitosa, Bistorta vivipara, Saxifraga сеrnua, bryophytes, $74^{\circ} 3^{\prime} 48.4^{\prime \prime} \mathrm{N}, 93^{\circ} 40^{\prime} 57.2^{\prime \prime} \mathrm{W}, 40$ m, 7 July 2013, P. Sokoloff 135 (CAN 603297); west ridge overlooking Gull Canyon, $4 \mathrm{~km}$ east of Arctic Watch Lodge, dry rocky scree, with Xanthoria elegans, Festuca sp., Saxifraga oppositifolia, 74³'42.4"N, $93^{\circ} 40^{\prime} 47.8^{\prime \prime} \mathrm{W}, 50 \mathrm{~m}, 7$ July 2013, P. Sokoloff 138 (CAN 603298); gravelly scree ledge above Cunningham River, directly adjacent to Arctic Watch Lodge (north side), rocky talus irrigated by water pipe, with
Draba corymbosa, $74^{\circ} 4^{\prime} 10.8^{\prime \prime} \mathrm{N}, 93^{\circ} 48^{\prime} 37.4^{\prime \prime} \mathrm{W}, 58 \mathrm{~m}$, 8 July 2013, P. Sokoloff 151 (CAN 603299); northfacing slope west of Arctic Watch Lodge, wet rocky seep in Dryas-Eriophorum tundra, with Dryas integrifolia, Salix arctica, Draba corymbosa, Cerastium arcticum, 74²'17.5"N, 9349'17.2"W, 16 m, 9 July 2013, P. Sokoloff 165 (CAN 603300); Flat Rock Falls, east coast of Cunningham Inlet, $4 \mathrm{~km}$ northeast of Arctic Watch Lodge, wet Dryas tundra with snow-bed community, with Saxifraga oppositifolia, Salix arctica, $74^{\circ} 5^{\prime} 56.1^{\prime \prime N}, 93^{\circ} 44^{\prime} 18.5^{\prime \prime} \mathrm{W}, 55 \mathrm{~m}, 10$ July $2013, P$. Sokoloff 210 (CAN 603301); gravelly scree ledge above Cunningham River, $9 \mathrm{~km}$ southeast of Arctic Watch Lodge, dry gravel and barren rocks, $73^{\circ} 59^{\prime} 27.3^{\prime \prime} \mathrm{N}$, $93^{\circ} 40^{\prime} 57.5^{\prime \prime W}, 69$ m, 11 July 2013, P. Sokoloff 234 (CAN 603302); garden spot below sewage lagoon at Arctic Watch Lodge, lush green patch in rocky scree, with Salix arctica, Saxifraga oppositifolia, Papaver sp., Parrya arctica, bryophytes, $74^{\circ} 4^{\prime} 13.1^{\prime \prime} \mathrm{N}, 93^{\circ} 48^{\prime} 55.8^{\prime \prime} \mathrm{W}$, 16 m, 12 July 2013, P. Sokoloff, A. Manik 245 (CAN 603303).

Draba glabella Pursh (Smooth Draba, drave glabre) [circumboreal-polar] - NunAvUT: Somerset Island, western cliff wall of Gull Canyon, below gull nesting area, wet rocks directly under water seeps and waterfall, dense lush vegetation, with Saxifraga cespitosa, Bistorta vivipara, Saxifraga cernua, bryophytes, $74^{\circ} 3^{\prime} 48.4^{\prime \prime} \mathrm{N}$,

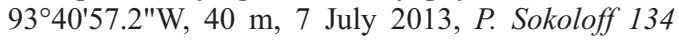
(CAN 603304).

Draba lactea Adams (Milky Draba, drave laiteuse) [circumpolar] - NunAVUT: Somerset Island, Cunningham Inlet, $1.6 \mathrm{~km}$ from inlet, uplands, polar desert, sedge meadow, $74^{\circ} 06^{\prime} \mathrm{N}, 93^{\circ} 55^{\prime} \mathrm{W}, 20 \mathrm{~m}, 22$ July 1976 , L.C. Bliss s.n. (ALTA 56712);

Draba nivalis Liljebkad (Snow Draba, drave des neiges) [circumpolar-alpine] - NuNAVUT: Somerset Island, south end of Sunday Lake, north of Cunningham River, $7 \mathrm{~km}$ south of Arctic Watch Lodge, lemming mound in middle of mud flats, $74^{\circ} 0^{\prime} 23.3^{\prime \prime} \mathrm{N}$, $93^{\circ} 42^{\prime} 43.5^{\prime \prime} \mathrm{W}, 41 \mathrm{~m}, 11$ July 2013, P. Sokoloff 228 (CAN 603305).

Draba simmonsii Elven \& Al-Shebaz (Simmons' Draba, drave de Simmons) [North American] - NunAvUT: Somerset Island, gravelly scree ledge above Cunningham River, directly adjacent to Arctic Watch Lodge (north side), rocky talus irrigated by water pipe, with Draba corymbosa, $74^{\circ} 4^{\prime} 10.8^{\prime \prime} \mathrm{N}, 93^{\circ} 48^{\prime} 37.4^{\prime \prime} \mathrm{W}, 58 \mathrm{~m}$, 8 July 2013, P. Sokoloff 152 (CAN 603306).

Draba subcapitata Simmons (Ellesmere Island Draba, drave subcapitée) [circumpolar] - NunAVUT: Somerset Island, scarce on dry gravel slope, $74^{\circ} 6^{\prime} \mathrm{N}$, $93^{\circ} 51^{\prime} \mathrm{W}, 11$ August 1958, D.B.O. Savile 3786 (DAO 525915).

Eutrema edwardsii R. Brown (Edwards' Mock Wallflower, eutréma d'Edwards) [circumpolar-alpine] NunAVUT: Somerset Island, Cunningham Inlet, $1.6 \mathrm{~km}$ from inlet, Stand 15, uplands, polar desert, sedge meadow, $74^{\circ} 06^{\prime} \mathrm{N}, 93^{\circ} 55^{\prime} \mathrm{W}, 20 \mathrm{~m}, 22$ July 1976, L.C. Bliss s.n. (ALTA 56711). 
Parrya arctica R. Brown (Arctic False Wallflower, parrya arctique) [North American] - NunAVUT: Somerset Island, Cunningham Inlet, $0.16 \mathrm{~km}$ from inlet, uplands, polar desert, coastal beach ridge, $74^{\circ} 06^{\prime} \mathrm{N}$, 935'ㄴ'W, 20 m, 23 July 1976, L.C. Bliss s.n. (ALTA 56729); Cunningham Inlet, on a mild slope, with Dryas integrifolia, $74^{\circ} 6^{\prime} 00^{\prime \prime} \mathrm{N}, 93^{\circ} 51^{\prime} 00^{\prime \prime} \mathrm{W}, 63 \mathrm{~m}, 13$ July 2004, A. Archambault \& L. Consaul aa 48 (CAN 603404), aa 49 (CAN 603403), aa52 (CAN 603402), aa 53 (CAN 603401), aa 55 (CAN 603399), aa 56 (CAN 603397), aa 57 (CAN 603398), aa59 (CAN 603405); scattered on dry calcareous gravel slope, $74^{\circ} 6^{\prime} \mathrm{N}$, $93^{\circ} 51^{\prime} \mathrm{W}, 11$ August 1958, D.B.O. Savile 3783 (DAO 567232); Cunningham Inlet, $74^{\circ} \mathrm{N}, 94^{\circ} \mathrm{W}, 7$ August 1949, B. Shindman s.n. (DAO 567235); west ridge overlooking Gull Canyon, $4 \mathrm{~km}$ east of Arctic Watch Lodge, tundra in dry mud, with Dryas integrifolia, $74^{\circ} 3^{\prime} 41.1^{\prime \prime} \mathrm{N}, 93^{\circ} 41^{\prime} 18.8^{\prime \prime} \mathrm{W}, 50 \mathrm{~m}, 7$ July 2013, $P$. Sokoloff 126 (CAN 603307); wet snowmelt valley at base of large unnamed mountain northwest of Arctic Watch Lodge, dry mud and clay mound in wet turfy tundra, with Festuca sp., 744'31.3"N, 93'51'1.7"W, 122 m, 9 July 2013, P. Sokoloff 180 (CAN 603308); wet snowmelt valley at base of large unnamed mountain northwest of Arctic Watch Lodge, wet mossy tundra, with Eriophorum angustifolium, $74^{\circ} 4^{\prime} 31.3^{\prime \prime} \mathrm{N}$, 935'ㄴ'1.7"W, 122 m, 9 July 2013, P. Sokoloff 185 (CAN 603309); south end of Sunday Lake, $7 \mathrm{~km}$ south of Arctic Watch Lodge, wet Eriophorum meadow, $74^{\circ} 0 ' 24.9^{\prime \prime} \mathrm{N}, 93^{\circ} 43^{\prime} 40.9^{\prime \prime} \mathrm{W}, 26 \mathrm{~m}, 11$ July $2013, P$. Sokoloff 224 (CAN 603310); shoreline on north coast of Somerset Island, $7 \mathrm{~km}$ west of entrance to Cunningham Inlet, rocky, snow-pack mountain, with Parry arctica, Saxifraga oppositifolia, Salix arctica, 74 $7^{\prime} 56.9^{\prime \prime} \mathrm{N}$, 94¹'21.8"W, 23 m, 11 July 2013, P. Sokoloff, Z. Halem 242 (CAN 603311).

\section{Caryophyllaceae}

Cerastium arcticum Lange (Arctic Chickweed, céraiste arctique, Nunarait qakuqtat) [North Americanamphi-Atlantic-European] - NunAVUT: Somerset Island, Cunningham Inlet, $8 \mathrm{~km}$ from inlet, uplands, polar desert, $74^{\circ} 06^{\prime} \mathrm{N}, 93^{\circ} 55^{\prime} \mathrm{W}, 200 \mathrm{~m}, 23$ July 1976, L.C. Bliss s.n. (ALTA 56720); west ridge overlooking Gull Canyon, $4 \mathrm{~km}$ east of Arctic Watch Lodge, dry rocky scree, with Xanthoria elegans, Festuca sp., Saxifraga oppositifolia, $74^{\circ} 3^{\prime} 42.4^{\prime \prime} \mathrm{N}, 93^{\circ} 40^{\prime} 47.8^{\prime \prime} \mathrm{W}, 50 \mathrm{~m}, 7$ July 2013, P. Sokoloff 131 (CAN 603312); confluence of Cunningham River and stream immediately south of Arctic Watch Lodge at Cunningham River crossing, rocky talus on south-facing slope, $74^{\circ} 4^{\prime} 2.9^{\prime \prime} \mathrm{N}$, 9348'31.7"W, 58 m, 8 July 2013, P. Sokoloff 148 (CAN 603313); gravelly scree ledge above Cunningham River, directly adjacent to Arctic Watch Lodge (north side), rocky talus irrigated by water pipe, with Draba corymbosa, $74^{\circ} 4^{\prime} 10.8^{\prime \prime} \mathrm{N}, 93^{\circ} 48^{\prime} 37.4^{\prime \prime} \mathrm{W}, 58 \mathrm{~m}$, 8 July 2013, P. Sokoloff 155 (CAN 603314).

Cerastium beeringianum Chamussi \& Schlechtendal (Bering Sea Chickweed, céraiste du détroit de Béring)
[Asian-amphi-Beringian-North American] - NuNAVUT: Somerset Island, occasional on slightly moist calcareous gravel slopes, $74^{\circ} 6^{\prime} \mathrm{N}, 93^{\circ} 51^{\prime} \mathrm{W}, 11$ August 1958 , D.B.O. Savile 3784 (DAO 562713).

Cerastium regelii Ostenfeld (Regel's Chickweed, céraiste de Regel) [circumpolar] - NunAvUT: Somerset Island, dry stone ridge immediately west of Arctic Watch Lodge, east-facing wet muddy snow bed set in shale rocks, with Luzula confusa, Sabulina rubella, 74²'21.3"N, 9350'30.7"W, 122 m, July 6, 2013, P. Sokoloff 119 (CAN 603315).

Sabulina rossii (R. Brown ex Richardson) Dillenberger \& Kadereit (Ross' Stitchwort, sabline de Ross) [amphi-Beringian-North American-amphi-Atlantic] - NunAVUT: Somerset Island, Cunningham Inlet, $8 \mathrm{~km}$ from inlet, uplands, polar desert, $74^{\circ} 06^{\prime} \mathrm{N}, 93^{\circ} 55^{\prime} \mathrm{W}$, 200 m, 23 July 1976, L.C. Bliss s.n. (ALTA 72149); north-facing ridge west of Arctic Watch Lodge, Eriophorum-Arctagrostis meadow, $74^{\circ} 4^{\prime} 17.5^{\prime \prime} \mathrm{N}$, 9349'17.2"W, 16 m, 9 July 2013, P. Sokoloff 169 (CAN 603316); Flat Rock Falls, east coast of Cunningham Inlet, $4 \mathrm{~km}$ northeast of Arctic Watch Lodge, wet snow-patch community at foot of packed snowbank, near foot of falls, $74^{\circ} 5^{\prime} 56.1^{\prime \prime} \mathrm{N}, 93^{\circ} 44^{\prime} 18.5^{\prime \prime} \mathrm{W}, 55 \mathrm{~m}$, 10 July 2013, P. Sokoloff 201 (CAN 603317).

Sabulina rubella (Wahlenberg) Dillenberger \& Kadereit (Reddish Stitchwort, sabline rougeâtre, Kakillarnait) [circumpolar-alpine] - NUNAVUT: Somerset Island, scarce on calcareous gravel slope, $74^{\circ} 6^{\prime} \mathrm{N}$, $93^{\circ} 51^{\prime} \mathrm{W}, 11$ August 1958, D.B.O. Savile 3787 (DAO 527824); Cunningham Inlet, $8 \mathrm{~km}$ from inlet, uplands, polar desert, $74^{\circ} 06^{\prime} \mathrm{N}, 93^{\circ} 55^{\prime} \mathrm{W}, 200 \mathrm{~m}, 20$ July 1976 , L.C. Bliss s.n. (ALTA 56728); dry stone ridge immediately west of Arctic Watch Lodge, east-facing wet muddy snow bed set in shale rocks, with Luzula confusa, $74^{\circ} 4^{\prime} 21.3^{\prime \prime N}, 93^{\circ} 50^{\prime} 30.7^{\prime \prime} \mathrm{W}, 122 \mathrm{~m}$, July 6, 2013, $P$. Sokoloff 118 (CAN 603318); Flat Rock Falls, east coast of Cunningham Inlet, $4 \mathrm{~km}$ northeast of Arctic Watch Lodge, wet snow-patch community at foot of packed snowbank, near foot of falls, $74^{\circ} 5^{\prime} 56.1^{\prime \prime} \mathrm{N}$, $93^{\circ} 44^{\prime} 18.5^{\prime \prime} \mathrm{W}, 55 \mathrm{~m}, 10$ July 2013, P. Sokoloff 202 (CAN 603319); south end of Sunday Lake, $6 \mathrm{~km}$ south of Arctic Watch Lodge, mossy knoll in wet tundra, $74^{\circ} 1^{\prime} 7.8^{\prime \prime} \mathrm{N}, 93^{\circ} 45^{\prime} 41.5^{\prime \prime} \mathrm{W}, 66 \mathrm{~m}, 11$ July 2013, $P$. Sokoloff 213 (CAN 603320); lemming mound at top of hill at south end of Sunday Lake, $7 \mathrm{~km}$ south of Arctic Watch Lodge, with Potentilla sp., Alopecurus magellanicus, $74^{\circ} 0^{\prime} 38.8^{\prime \prime} \mathrm{N}, 93^{\circ} 44^{\prime} 30.4^{\prime \prime} \mathrm{W}, 49 \mathrm{~m}, 11$ July 2013, P. Sokoloff 219 (CAN 603321).

Silene uralensis (Ruprecht) Bocquet ssp. uralensis (Nodding Catchfly, silène de l'Oural, Pulluliujuit) [European-Asian-amphi-Beringian-North American] - NunAVut: Somerset Island, scattered on wet gravelly slope, $74^{\circ} 6^{\prime} \mathrm{N}, 93^{\circ} 51^{\prime} \mathrm{W}, 11$ August 1958, D.B.O. Savile 3781 (DAO 537745); gravelly scree ledge above Cunningham River, directly adjacent of Arctic Watch Lodge (north side), rocky talus irrigated by water pipe, with Draba corymbosa, $74^{\circ} 4^{\prime} 10.8^{\prime \prime} \mathrm{N}, 93^{\circ} 48^{\prime} 37.4^{\prime \prime} \mathrm{W}$, 58 m, 8 July 2013, P. Sokoloff 153 (CAN 603322). 
Stellaria longipes Goldie (Long-stalked Starwort, stellaire à longs pédicelles, Miqqaviat) [circumborealpolar] - NunAVUT: Somerset Island, confluence of Cunningham River and stream immediately south of Arctic Watch Lodge at Cunningham River crossing, rocky talus on south-facing slope, with Cerastium arcticum, $74^{\circ} 4^{\prime} 2.9^{\prime \prime} \mathrm{N}, 93^{\circ} 48^{\prime} 31.7^{\prime \prime} \mathrm{W}, 58 \mathrm{~m}, 8$ July 2013 , P. Sokoloff 145 (CAN 603323); wet snowmelt valley at base of large unnamed mountain northwest of Arctic Watch Lodge, dry mud and clay mound in wet turfy tundra, with Festuca sp., 74² $4^{\prime} 31.3^{\prime \prime N}, 93^{\circ} 51^{\prime} 1.7^{\prime \prime W}$, 122 m, 9 July 2013, P. Sokoloff 182 (CAN 603324, NFM); gravelly scree ledge above Cunningham River, $9.6 \mathrm{~km}$ southeast of Arctic Watch Lodge, muddy bank, 7359'27.3"N, 934ㅇ'57.5"W, 69 m, 11 July 2013, P. Sokoloff 235 (CAN 603325, US); gravelly scree ledge above Cunningham River, $9.6 \mathrm{~km}$ southeast of Arctic Watch Lodge, muddy bank, $73^{\circ} 59^{\prime} 27.3^{\prime \prime N}$, $93^{\circ} 40^{\prime} 57.5^{\prime \prime} \mathrm{W}, 69 \mathrm{~m}, 11$ July 2013, P. Sokoloff 236 (CAN 603326).

\section{Cyperaceae}

Carex aquatilis var. minor Boott (Arctic Water Sedge, carex mineur, Kilirnait) [circumboreal-polar] - NuNAVUT: Somerset Island, south end of Sunday Lake, $7 \mathrm{~km}$ south of Arctic Watch Lodge, wet Eriophorum meadow, $74^{\circ} 0^{\prime} 24.9^{\prime \prime} \mathrm{N}, 93^{\circ} 43^{\prime} 40.9^{\prime \prime} \mathrm{W}, 26 \mathrm{~m}, 11$ July 2013, P. Sokoloff 244 (CAN 603328).

Carex capillaris ssp. fuscidula (V.I. Kreczetovicz ex T.V. Egorova) Á. Löve \& D. Löve (Dusky-spike Sedge, carex à epis sombres) [circumpolar-alpine] - NUNAVUT: Somerset Island, Cunningham Inlet, $1.6 \mathrm{~km}$ from inlet, Stand 15 , uplands, polar desert, sedge meadow, $74^{\circ} 06^{\prime} \mathrm{N}$, $93^{\circ} 55^{\prime} \mathrm{W}, 20 \mathrm{~m}, 22$ July 1976, L.C. Bliss s.n. (ALTA 56726); north-facing slope west of Arctic Watch Lodge, wet rocky seep in Dryas-Eriophorum tundra, with Dryas integrifolia, Salix arctica, Draba corymbosa, Cerastium arcticum, $74^{\circ} 4^{\prime} 17.5^{\prime \prime} \mathrm{N}, 9^{\circ} 49^{\prime} 17.2^{\prime \prime} \mathrm{W}, 16 \mathrm{~m}$, 9 July 2013, P. Sokoloff 168 (CAN 603329); Flat Rock Falls, east coast of Cunningham Inlet, $4 \mathrm{~km}$ northeast of Arctic Watch Lodge, wet Dryas tundra with snowbed community, with Saxifraga oppositifolia, Salix arctica, $74^{\circ} 5^{\prime} 56.1^{\prime \prime N}, 93^{\circ} 44^{\prime} 18.5^{\prime \prime} \mathrm{W}, 55 \mathrm{~m}, 10$ July 2013, P. Sokoloff 207 (CAN 603330).

Carex membranacea Hooker (Fragile Sedge, carex membraneux, Kilirnait ajjikasangit iviit) [amphiBeringian-North American] - NuNAVUT: Somerset Island, scattered in small sedge meadow below limestone hill, $74^{\circ} 6^{\prime} \mathrm{N}, 93^{\circ} 51^{\prime} \mathrm{W}, 11$ August 1958, D.B.O. Savile 3777 (DAO 363708).

Eriophorum triste (Th. Fries) Hadač \& Á. Löve (Tall Cottongrass, linaigrette triste) [amphi-Beringian-North American-amphi-Atlantic] - NunAVUT: Somerset Island, Cunningham Inlet, $1.6 \mathrm{~km}$ from inlet, wet sedge tundra, sedge meadow, $74^{\circ} 06^{\prime} \mathrm{N}, 93^{\circ} 55^{\prime} \mathrm{W}, 20 \mathrm{~m}, 22$ July 1976, L.C. Bliss s.n. (ALTA 56715); North-facing ridge west of Arctic Watch Lodge, Eriophorum-Arctagrostis meadow, $74^{\circ} 4^{\prime} 17.5^{\prime \prime} \mathrm{N}, 93^{\circ} 49^{\prime} 17.2^{\prime \prime} \mathrm{W}, 16 \mathrm{~m}, 9$ July 2013, P. Sokoloff 170 (CAN 603335, US); south end of Sunday Lake, $7 \mathrm{~km}$ south of Arctic Watch Lodge, wet Eriophorum meadow, $74^{\circ} 00^{\prime} 24.9^{\prime \prime} \mathrm{N}, 93^{\circ} 43^{\prime} 40.9^{\prime \prime} \mathrm{W}$, 26 m, 11 July 2013, P. Sokoloff 222 (CAN 603336); south end of Sunday Lake, $7 \mathrm{~km}$ south of Arctic Watch Lodge, wet Eriophorum meadow, $74^{\circ} 0 ' 24.9^{\prime \prime} \mathrm{N}$, $93^{\circ} 43^{\prime} 40.9^{\prime \prime} \mathrm{W}, 26$ m, 11 July 2013, P. Sokoloff 223 (CAN 603337).

\section{Juncaceae}

Juncus biglumis L. (Two-flowered Rush, jonc à deux glumes, Iviit) [circumpolar-alpine] - NunAVUT: Somerset Island, occasional on wet calcareous slopes, $74^{\circ} 6^{\prime} \mathrm{N}, 93^{\circ} 51^{\prime} \mathrm{W}, 11$ August 1958, D.B.O. Savile 3778 (DAO 781829); Cunningham Inlet, $1.6 \mathrm{~km}$ from inlet, wet sedge tundra, coastal lowland beach ridges, $74^{\circ} 06^{\prime} \mathrm{N}, 93^{\circ} 55^{\prime} \mathrm{W}, 20 \mathrm{~m}, 23$ July 1976, L.C. Bliss s.n. (ALTA 56721); north-facing slope west of Arctic Watch Lodge, wet rocky seep in Dryas-Eriophorum tundra, with Dryas integrifolia, Salix arctica, Draba corymbosa, Cerastium arcticum, $74^{\circ} 4^{\prime} 17.5^{\prime \prime} \mathrm{N}, 93^{\circ} 49^{\prime} 17.2^{\prime \prime} \mathrm{W}, 16$ m, 9 July 2013, P. Sokoloff 166 (CAN 603341, US); wet snowmelt valley at base of large unnamed mountain northwest of Arctic Watch Lodge, wet mossy tundra, with Eriophorum angustifolium, $74^{\circ} 4^{\prime} 31.3^{\prime \prime} \mathrm{N}$, 935'ㄴ'1"W, 122 m, 9 July 2013, P. Sokoloff 183 (CAN 603342).

Luzula nivalis (Laestadius) Sprengel (Arctic Woodrush, luzule arctique) [circumpolar-alpine] - NunAVUT: Somerset Island, scattered on moist calcareous slopes, $74^{\circ} 6^{\prime} \mathrm{N}, 93^{\circ} 51^{\prime} \mathrm{W}, 11$ August 1958, D.B.O. Savile 3782 (DAO 780607); dry stone ridge immediately west of Arctic Watch Lodge, east-facing wet muddy snow bed set in shale rocks, with Sabulina rubella, $74^{\circ} 4^{\prime} 21.3^{\prime \prime} \mathrm{N}$, 9350'30.7"W, 122 m, July 6, 2013, P. Sokoloff 117 (CAN 603343); north-facing slope west of Arctic Watch Lodge, wet rocky seep in Dryas-Eriophorum tundra, with Dryas integrifolia, Salix arctica, Draba corymbosa, Cerastium arcticum, 74²'17.5"N, 9349'17.2"W, 16 m, 9 July 2013, P. Sokoloff 167 (CAN 603344); Flat Rock Falls, east coast of Cunningham Inlet, $4 \mathrm{~km}$ northeast of Arctic Watch Lodge, wet snow-patch community at foot of packed snowbank, near foot of falls, $74^{\circ} 5^{\prime} 56.1^{\prime \prime N}, 93^{\circ} 44^{\prime} 18.5^{\prime \prime} \mathrm{W}, 55 \mathrm{~m}, 10$ July 2013, $P$. Sokoloff 199 (CAN 603345, US).

\section{Orobanchaceae}

Pedicularis lanata Willdenow ex Chamisso \& Schlechtendal (Woolly Lousewort, pédiculaire laineuse, Ugjungnaq) [amphi-Beringian-North American] NunAVUT: Somerset Island, south end of Sunday Lake, $6 \mathrm{~km}$ south of Arctic Watch Lodge, wet SalixDryas meadow, $74^{\circ} 0^{\prime} 51.7^{\prime \prime} \mathrm{N}, 93^{\circ} 44^{\prime} 57.6^{\prime \prime} \mathrm{W}, 64 \mathrm{~m}$, 11 July 2013, P. Sokoloff 216 (CAN 603347); south end of Sunday Lake, $7 \mathrm{~km}$ south of Arctic Watch Lodge, wet Eriophorum meadow, $74^{\circ} 00^{\prime} 24.9^{\prime \prime} \mathrm{N}, 93^{\circ} 43^{\prime} 40.9^{\prime \prime} \mathrm{W}$, 26 m, 11 July 2013, P. Sokoloff 225 (CAN 603348).

\section{Papaveraceae}

Papaver cornwallisense D. Löve (Cornwallis Island Poppy, pavot de Cornwallis) [North American-amphi- 
Atlantic] - NunAVUT: Somerset Island, Cunningham Inlet, $0.16 \mathrm{~km}$ from inlet, uplands, polar desert, $74^{\circ} 06^{\prime} \mathrm{N}$, 935'' 20 m, 23 July 1976, L.C. Bliss s.n. (ALTA 56725); Cunningham Inlet, $0.16 \mathrm{~km}$ from inlet, uplands, polar desert, $74^{\circ} 06^{\prime} \mathrm{N}, 93^{\circ} 55^{\prime} \mathrm{W}, 20 \mathrm{~m}, 23$ July 1976, L.C. Bliss s.n. (ALTA 56710); dry stone ridge immediately west of Arctic Watch Lodge, in gravel scree, dry slaty gravel, with Saxifraga oppositifolia, Salix arctica, Papaver sp., 74²'20.2"N, 934'ㄹ' $22.6^{\prime \prime} \mathrm{W}$, 20 m, July 6, 2013, P. Sokoloff 115 (CAN 603349); western cliff wall of Gull Canyon, below Gull nesting area, wet rocks directly under water seeps and waterfall, dense lush vegetation, with Saxifraga cespitosa, Bistorta vivipara, Saxifraga cernua, bryophytes, $74^{\circ} 3^{\prime} 48.4^{\prime \prime} \mathrm{N}$, $93^{\circ} 40^{\prime} 57.2^{\prime \prime}, 40 \mathrm{~m}, 7$ July 2013 , P. Sokoloff 136 (CAN 603350); gravelly scree ledge above Cunningham River, directly adjacent to Arctic Watch Lodge (north side), rocky talus irrigated by water pipe, with Draba corymbosa, $74^{\circ} 4^{\prime} 10.8^{\prime \prime} \mathrm{N}, 93^{\circ} 48^{\prime} 37.4^{\prime \prime} \mathrm{W}, 58 \mathrm{~m}$, 8 July 2013, P. Sokoloff 150 (CAN 603351); south end of Sunday Lake, north of Cunningham River, $7 \mathrm{~km}$ south of Arctic Watch Lodge, mud flats, $74^{\circ} 0^{\prime} 23.3^{\prime \prime} \mathrm{N}$, $93^{\circ} 42^{\prime} 43.5^{\prime \prime} \mathrm{W}, 41 \mathrm{~m}, 11$ July 2013, P. Sokoloff 231 (CAN 603352).

\section{Poaceae}

Alopecurus magellanicus Lamarck (Alpine Foxtail, vulpin boréal, Ivi) [circumpolar-alpine and South American] - NunAVUT: Somerset Island, site no. Z-25, level lacustrine well- to imperfectly drained silt plain, grass-saxifrage foxhole mound, $74^{\circ} 2^{\prime} \mathrm{N}, 93^{\circ} 30^{\prime} \mathrm{W}, 53 \mathrm{~m}$, 260 m, July 1, 1975, S.C. Zoltai 751152 (DAO 137589); mound at top of hill at south end of Sunday Lake, $7 \mathrm{~km}$ south of Arctic Watch Lodge, lush lemming mound, with Potentilla sp., Sabulina rubella, $74^{\circ} 0^{\prime} 38.8^{\prime \prime}$, 934' 30.4"W, 49 m, 11 July 2013, P. Sokoloff 218 (CAN 603353); south end of Sunday Lake, north of Cunningham River, $7 \mathrm{~km}$ south of Arctic Watch Lodge, lemming mound in middle of mud flats, $74^{\circ} 0^{\prime} 23.3^{\prime \prime} \mathrm{N}$,

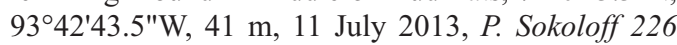
(CAN 603354); confluence of Cunningham River and stream immediately south of Arctic Watch Lodge at Cunningham River crossing, rocky talus on southfacing slope, with bryophytes, Cerastium arcticum, $74^{\circ} 4^{\prime 2} .9^{\prime \prime N}, 93^{\circ} 48^{\prime} 31.7^{\prime \prime} \mathrm{W}, 58 \mathrm{~m}, 8$ July 2013, . Sokoloff 143b (CAN 603355).

Arctagrostis latifolia (R. Brown) Grisebach ssp. latifolia (Polargrass, arctagrostide à larges feuilles) [circumpolar-alpine] - NunAVUT: Somerset Island, Cunningham Inlet, $1.6 \mathrm{~km}$ from inlet, wet sedge tundra on coastal lowlands, $74^{\circ} 06^{\prime} \mathrm{N}, 93^{\circ} 55^{\prime} \mathrm{W}, 20 \mathrm{~m}, 23$ July 1976, L.C. Bliss s.n., (ALTA 56714); alluvial plain of Cunningham River on Cunningham Inlet, $3 \mathrm{~km}$ east of Arctic Watch Lodge, wet sedge meadow emerging from melting snowbank, with Deschampsia sp., Poa sp., $74^{\circ} 3^{\prime} 40.1^{\prime \prime} \mathrm{N}, 93^{\circ} 42^{\prime} 17.6^{\prime \prime} \mathrm{W}, 40 \mathrm{~m}, 7$ July 2013, $P$. Sokoloff 121 (CAN 603356); north-facing ridge west of Arctic Watch Lodge, Eriophorum-Arctagrostis meadow, $74^{\circ} 4^{\prime} 17.5^{\prime \prime} \mathrm{N}, 93^{\circ} 49^{\prime} 17.2^{\prime \prime} \mathrm{W}, 16 \mathrm{~m}, 9$ July 2013, P. Sokoloff 172 (CAN 603357).

Arctophila fulva (Trinius) Andersson (Pendant Grass, arctophile fauve) [circumpolar] - NUNAVUT: Somerset Island, site no. Z-44, level fen with poorly drained organic soil, with Drepanocladus sp., $74^{\circ} 1^{\prime} \mathrm{N}, 93^{\circ} 27^{\prime} \mathrm{W}$, 53 m, 14 July 1975, S. C. Zoltai 751114 (DAO 137672).

Deschampsia brevifolia R. Brown (Short-leaved Hairgrass, deschampsie à feuilles courtes) [Asianamphi-Beringian-North American] - NunAVUT: Somerset Island, alluvial plain of Cunningham River on Cunningham Inlet, $3 \mathrm{~km}$ east of Arctic Watch Lodge, wet sedge meadow emerging from melting snowbank, with Poa sp., Arctagrostis sp., $74^{\circ} 3^{\prime} 40.1^{\prime \prime N}$, 9342'17.6"W, 40 m, 7 July 2013, P. Sokoloff 122 (CAN 603358); alluvial plain of Cunningham River on Cunningham Inlet, $3 \mathrm{~km}$ east of Arctic Watch Lodge, wet sedge meadow emerging from melting snowbank, with Poa sp., Arctagrostis sp., $74^{\circ} 3^{\prime} 40.1^{\prime \prime N}, 93^{\circ} 42^{\prime} 17.6^{\prime \prime} \mathrm{W}, 40 \mathrm{~m}, 7$ July $2013, P$. Sokoloff 123 (CAN 603359); north-facing ridge west of Arctic Watch Lodge, Eriophorum-Arctagrostis meadow, $74^{\circ} 4^{\prime} 17.5^{\prime \prime} \mathrm{N}, 93^{\circ} 49^{\prime} 17.2^{\prime \prime} \mathrm{W}, 16 \mathrm{~m}, 9$ July 2013, P. Sokoloff 173 (CAN 603360, US); south end of Sunday Lake, $6 \mathrm{~km}$ south of Arctic Watch Lodge, mossy knoll in wet tundra, $74^{\circ} 11^{\prime} 7.8^{\prime \prime} \mathrm{N}, 93^{\circ} 45^{\prime} 41.5^{\prime \prime} \mathrm{W}$, 66 m, 11 July 2013, P. Sokoloff 215 (CAN 603361).

Festuca baffinensis Polunin (Baffin Island Fescue, fétuque de Baffin) [Asian-amphi-Beringian-North American-amphi-Atlantic] - NunAVuT: Somerset Island, site no. Z-25, level lacustrine well- to imperfectly drained silt plain, grass-saxifrage foxhole mound, $74^{\circ} 2^{\prime} \mathrm{N}, 93^{\circ} 30^{\prime} \mathrm{W}, 53 \mathrm{~m}, 1$ July $1975, S$. $C$. Zoltai 751153 (DAO 137687).

Festuca brachyphylla Schultes \& Schultes f. (Shortleaved Fescue, fétuque à feuilles courtes, Ivilsugait) [circumpolar-alpine] - NuNAVUT: Somerset Island, confluence of Cunningham River and stream immediately south of Arctic Watch Lodge at Cunningham River crossing, rocky talus on south-facing slope, with bryophytes, Cerastium arcticum, $74^{\circ} 4^{\prime} 2.9^{\prime \prime} \mathrm{N}, 9^{\circ} 48^{\prime} 31.7^{\prime \prime} \mathrm{W}$, 58 m, 8 July 2013, P. Sokoloff 146 (CAN 603362).

Festuca edlundiae S.G. Aiken, Consaul \& Lefkovitch (Edlund's Fescue, fétuque d'Edlund) [amphiBeringian-North American-amphi-Atlantic] NuNAVUT: Somerset Island, west ridge overlooking Gull Canyon, $4 \mathrm{~km}$ east of Arctic Watch Lodge, dry rocky scree, with Xanthoria elegans, Saxifraga oppositifolia, 74²'42.4"N, 9340'47.8"W, 50 m, 7 July 2013, P. Sokoloff 128 (CAN 603363).

Poa abbreviata R. Brown ssp. abbreviata (Dwarf Bluegrass, pâturin court) [nearly circumpolar] NunAVUT: Somerset Island, scarce on moist calcareous slope, $74^{\circ} 6^{\prime} \mathrm{N}, 93^{\circ} 51^{\prime} \mathrm{W}, 11$ August 1958, D.B.O. Savile 3780 (DAO 57589); Cunningham Inlet, 8 km from inlet, uplands, polar desert, $74^{\circ} 06^{\prime} \mathrm{N}, 93^{\circ} 55^{\prime} \mathrm{W}$, 225 m, 20 July 1976, L.C. Bliss s.n., (ALTA 56727); Cunningham Inlet, $8 \mathrm{~km}$ from inlet, uplands, polar 
desert, coastal lowlands, $74^{\circ} 06^{\prime} \mathrm{N}, 93^{\circ} 55^{\prime} \mathrm{W}, 30 \mathrm{~m}, 23$ July 1976, L.C. Bliss s.n., (ALTA 56716).

Poa arctica R. Brown (Arctic Bluegrass, pâturin arctique) [circumpolar-alpine] - NUNAVUT: Somerset Island, Flat Rock Falls, east coast of Cunningham Inlet, $4 \mathrm{~km}$ northeast of Arctic Watch Lodge, wet snow-patch community at foot of packed snowbank, near foot of falls, $74^{\circ} 5^{\prime} 56.1^{\prime \prime} \mathrm{N}, 93^{\circ} 44^{\prime} 18.5^{\prime \prime} \mathrm{W}, 55 \mathrm{~m}, 10$ July 2013 , P. Sokoloff 198 (CAN 603364).

Puccinellia bruggemannii T.J. Sørensen (Prince Patrick Alkaligrass, puccinellie de Bruggemann) [North American] - NunAVUT: Somerset Island, Cunningham Inlet, $1.6 \mathrm{~km}$ from inlet, uplands, polar desert, sedge meadow, $74^{\circ} 06^{\prime} \mathrm{N}, 93^{\circ} 55^{\prime} \mathrm{W}, 20 \mathrm{~m}, 22$ July $1976, L . C$. Bliss s.n., (ALTA 56719); on wet gravel and clay soil, $74^{\circ} 6^{\prime} 00^{\prime \prime N}, 93^{\circ} 51^{\prime} 00^{\prime \prime} \mathrm{W}, 63 \mathrm{~m}$, July 13, 2004, L. Consaul \& A. Archambault 3083 (CAN 603396).

Puccinellia vahliana (Liebmann) Scribner \& Merrill (Vahl's Alkaligrass, puccinellie de Vahl) [North American-amphi-Atlantic] - NunAvUT: Somerset Island, on wet gravel and clay soil, $74^{\circ} 6^{\prime} 00^{\prime \prime} \mathrm{N}$, 9351'00"W, 63 m, July 13, 2004, L. Consaul \& A. Archambault 3082 (CAN 603395); north-facing slope west of Arctic Watch Lodge, wet rocky seep in DryasEriophorum tundra, with Dryas integrifolia, Salix arctica, Draba corymbosa, Cerastium arcticum, $74^{\circ} 4^{\prime} 17.5^{\prime \prime} \mathrm{N}, 93^{\circ} 49^{\prime} 17.2^{\prime \prime} \mathrm{W}, 16 \mathrm{~m}, 9$ July 2013, P. Sokoloff 189 (CAN 603366).

Trisetum spicatum (L.) K. Richter (Narrow False Oats, trisète à epi, Iviit iviksugait) [circumpolaralpine] - NunAVUT: Somerset Island, confluence of Cunningham River and stream immediately south of Arctic Watch Lodge at Cunningham River crossing, rocky talus on south-facing slope, with bryophytes, Cerastium arcticum, $74^{\circ} 4^{\prime} 2.9^{\prime \prime} \mathrm{N}, 9^{\circ} 48^{\prime} 31.7^{\prime \prime} \mathrm{W}, 58 \mathrm{~m}$, 8 July 2013, P. Sokoloff 144 (CAN 603370).

\section{Polygonaceae}

Bistorta vivipara (L.) Delarbre (Alpine Bistort, renouée vivipare, Sapangaralannguat) [circumborealpolar] - NUNAVUT: Somerset Island, Cunningham Inlet, $1.6 \mathrm{~km}$ from inlet, uplands, polar desert, sedge meadow, $74^{\circ} 06^{\prime} \mathrm{N}, 93^{\circ} 55^{\prime} \mathrm{W}, 20 \mathrm{~m}, 22$ July 1976, L.C. Bliss s.n. (ALTA 56713); Flat Rock Falls, east coast of Cunningham Inlet, $4 \mathrm{~km}$ northeast of Arctic Watch Lodge, wet Dryas tundra with snow-bed community, with Saxifraga oppositifolia, Salix arctica, $74^{\circ} 5^{\prime} 56.1 " \mathrm{~N}$, 93044'18.5"W, 55 m, 10 July 2013, P. Sokoloff 209 (CAN 603371).

Oxyria digyna (L.) Hill (Mountain Sorrel, oxyrie de montagne, Qunguliit) [circumpolar-alpine] - NUNAVUT: Somerset Island, south end of Sunday Lake, $6 \mathrm{~km}$ south of Arctic Watch Lodge, mossy knoll in wet tundra, $74^{\circ} 17.8^{\prime \prime} \mathrm{N}, 93^{\circ} 45^{\prime} 41.5^{\prime \prime} \mathrm{W}, 66 \mathrm{~m}, 11$ July 2013, $P$. Sokoloff 212 (CAN 603372); mud flats on alluvial slope to Cunningham River, south of Sunday lake, $9 \mathrm{~km}$ south of Arctic Watch Lodge, mud flats alongside river, $73^{\circ} 59^{\prime} 16.5^{\prime \prime} \mathrm{N}, 93^{\circ} 41^{\prime} 38.7^{\prime \prime} \mathrm{W}, 40 \mathrm{~m}, 11$ July 2013, $P$. Sokoloff 237 (CAN 603373).

\section{Rosaceae}

Dryas integrifolia Vahl (Entire-leaved Mountain Avens, dryade à feuilles entières, Malikkaat) [amphiBeringian-North American] - NunAVUT: Somerset Island, Cunningham Inlet, $0.16 \mathrm{~km}$ from inlet, uplands, polar desert, coastal lowland beach ridges, $74^{\circ} 06^{\prime} \mathrm{N}$, $93^{\circ} 55^{\prime} \mathrm{W}, 30 \mathrm{~m}, 23$ July 1976, L.C. Bliss s.n. (ALTA 56730); north-facing ridge west of Arctic Watch Lodge, Eriophorum-Arctagrostis meadow, 74²'27.7"N, 9350'39.4"W, 118 m, 9 July 2013, P. Sokoloff 174 (CAN 603374); $2 \mathrm{~km}$ south of point at Cape Anne, muddy wet ground in marshy field alongside river, with Saxifraga oppositifolia, Salix arctica, Dryas integrifolia, bryophytes, $74^{\circ} 66^{\prime} 23.3^{\prime \prime} \mathrm{N}, 94^{\circ} 23^{\prime} 44.25^{\prime \prime} \mathrm{W}, 26 \mathrm{~m}$, 10 July 2013, P. Sokoloff, Z. Halem 240 (CAN 603375).

Potentilla pulchella R. Brown (Pretty Cinquefoil, potentille jolie) [circumpolar] - NunAVUT: Somerset Island, lemming mound at top of hill at south end of Sunday Lake, $7 \mathrm{~km}$ south of Arctic Watch Lodge, lush lemming mound, with Arctagrostis sp., Sabulina rubella, $74^{\circ} 0^{\prime} 38.8^{\prime \prime} \mathrm{N}, 93^{\circ} 44^{\prime} 30.4^{\prime \prime} \mathrm{W}, 49 \mathrm{~m}, 11$ July 2013 , P. Sokoloff 217 (CAN 603376); south end of Sunday Lake, north of Cunningham River, $7 \mathrm{~km}$ south of Arctic Watch Lodge, lemming mound in middle of mud flats, $74^{\circ} 0$ ' $23.3^{\prime \prime} \mathrm{N}, 93^{\circ} 42^{\prime} 43.5^{\prime \prime} \mathrm{W}, 41 \mathrm{~m}, 11$ July 2013 , P. Sokoloff 227 (CAN 603377).

\section{Salicaceae}

Salix arctica Pallas (Arctic Willow, saule arctique, Suputiit, Suputiksaliit, Uqaujait) [circumpolar-alpine] - Nunavut: Somerset Island, Cunningham Inlet, $1.6 \mathrm{~km}$ from inlet, uplands, polar desert, coastal lowland beach ridges, $74^{\circ} 06^{\prime} \mathrm{N}, 93^{\circ} 55^{\prime} \mathrm{W}, 30 \mathrm{~m}, 23$ July $1976, L$. C. Bliss s.n. (ALTA 56718); dry stone ridge immediately west of Arctic Watch Lodge, in gravel scree, dry slaty gravel, with Saxifraga oppositifolia, Salix arctica, Papaver sp., 744'20.2"N, 9349'22.6"W, 20 m, July 6, 2013, P. Sokoloff 113 (CAN 603379); dry stone ridge immediately west of Arctic Watch Lodge, in gravel scree, dry slaty gravel, with Saxifraga oppositifolia, Salix arctica, Papaver sp., 744'20.2"N, 9349'22.6"W, 20 m, July 6, 2013, P. Sokoloff 114 (CAN 603380); gravelly scree ledge above Cunningham River, directly adjacent to Arctic Watch Lodge (north side), rocky talus irrigated by water pipe, with Draba corymbosa, $74^{\circ} 4^{\prime} 10.8^{\prime \prime} \mathrm{N}, 93^{\circ} 48^{\prime} 37.4^{\prime \prime} \mathrm{W}, 58 \mathrm{~m}, 9$ July $2013, P$. Sokoloff 164 (CAN 603381); wet snowmelt valley at base of large unnamed mountain northwest of Arctic Watch Lodge, wet sedge meadow emerging from melting snowbank, with Stellaria longipes, Cerastium arcticum, bryophytes, $74^{\circ} 4^{\prime} 31.3^{\prime \prime} \mathrm{N}, 93^{\circ} 51^{\prime} 1.7^{\prime \prime} \mathrm{W}, 122 \mathrm{~m}$, 9 July 2013, P. Sokoloff 178 (CAN 603382); garden spot below sewage lagoon at Arctic Watch Lodge, lush green patch in rocky scree, with Salix arctica, Saxifraga oppositifolia, Papaver sp., Parrya arctica, bryophytes, $74^{\circ} 4^{\prime} 13.1^{\prime \prime N}, 93^{\circ} 48^{\prime} 55.8^{\prime \prime} \mathrm{W}, 16 \mathrm{~m}, 9$ July 2013 , P. Sokoloff 188 (CAN 603383); $2 \mathrm{~km}$ south of point at Cape Anne, muddy wet ground in marshy field alongside river, with Saxifraga oppositifolia, Salix arctica, 
Dryas integrifolia, bryophytes, $74^{\circ} 6^{\prime} 23.3^{\prime \prime} \mathrm{N}$, 94²3'44.25"W, 26 m, 10 July 2013, P. Sokoloff, Z. Halem 238 (CAN 603384); garden spot below sewage lagoon at Arctic Watch Lodge, lush green patch in rocky scree, with Salix arctica, Saxifraga oppositifolia, Papaver sp., Parrya arctica, bryophytes, $74^{\circ} 4^{\prime} 13.1^{\prime \prime N}$, 934'ㄷ․ㄹ"W, 16 m, July 12, 2013, P. Sokoloff, A. Manik 243 (CAN 603385).

\section{Saxifragaceae}

Micranthes nivalis (L.) Small (Snow Saxifrage, saxifrage des neiges) [circumpolar-alpine] - NunAVUT: Somerset Island, Flat Rock Falls, east coast of Cunningham Inlet, $4 \mathrm{~km}$ northeast of Arctic Watch Lodge, wet snow-patch community at foot of packed snowbank, near foot of falls, $74^{\circ} 5^{\prime} 56.1^{\prime \prime} \mathrm{N}, 93^{\circ} 44^{\prime} 18.5^{\prime \prime} \mathrm{W}$, 55 m, 10 July 2013, P. Sokoloff 203 (CAN 603386).

Saxifraga cernua L. (Nodding Saxifrage, saxifrage penchée, Nunaraq qupanuap niqinga) [circumpolaralpine] - NuNAVUT: Somerset Island, western cliff wall of Gull Canyon, below Gull nesting area, wet rocks directly under water seeps and waterfall, dense lush vegetation, with Saxifraga cespitosa, Bistorta vivipara, bryophytes, $74^{\circ} 3^{\prime} 48.4^{\prime \prime} \mathrm{N}, 93^{\circ} 40^{\prime} 57.2^{\prime \prime} \mathrm{W}, 40 \mathrm{~m}$, 7 July 2013, P. Sokoloff 133 (CAN 603387); gravelly scree ledge above Cunningham River, directly adjacent to Arctic Watch Lodge (north side), rocky talus irrigated by water pipe, with Draba corymbosa, $74^{\circ} 4^{\prime} 10.8^{\prime \prime N}$,

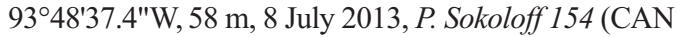
603388).

Saxifraga cespitosa L. (Tufted Saxifrage, saxifrage cespiteuse) [circumpolar-alpine] - NuNAVUT: Somerset Island, Cunningham Inlet, $0.16 \mathrm{~km}$ from inlet, uplands, polar desert, coastal lowland beach ridges, $74^{\circ} 06^{\prime} \mathrm{N}$, $93^{\circ} 55^{\prime} \mathrm{W}, 30 \mathrm{~m}, 23$ July 1976, L.C. Bliss s.n. (ALTA 56722); dry stone ridge immediately west of Arctic Watch Lodge, in gravel scree, wet seep under boulder in shale and muddy rock, with Cerastium arcticum, Papaver sp., Saxifraga oppositifolia, 74²'21.3"N, 9350'30.7"W, 122 m, July 6, 2013, P. Sokoloff 116 (CAN 603389, NFM); Flat Rock Falls, east coast of Cunningham Inlet, $4 \mathrm{~km}$ northeast of Arctic Watch Lodge, wet snow-patch community at foot of packed snowbank, near foot of falls, $74^{\circ} 5^{\prime} 56.1^{\prime \prime} \mathrm{N}, 93^{\circ} 44^{\prime} 18.5^{\prime \prime} \mathrm{W}$, 55 m, 10 July 2013, P. Sokoloff 200 (CAN 603390); garden spot below sewage lagoon at Arctic Watch Lodge, lush green patch in rocky scree, with Salix arctica, Saxifraga oppositifolia, Papaver sp., Parrya arctica, bryophytes, $74^{\circ} 4^{\prime} 13.1^{\prime \prime N}, 93^{\circ} 48^{\prime} 55.8^{\prime \prime} \mathrm{W}, 16 \mathrm{~m}$, July 12 , 2013, P. Sokoloff 246 (CAN 603391).

Saxifraga oppositifolia L. (Purple Mountain Saxifrage, saxifrage à feuilles opposées, Aupilattunnguat) [circumpolar-alpine] - NUNAVUT: Somerset Island, common especially on moist calcareous gravel slopes., $74^{\circ} 6^{\prime} \mathrm{N}, 93^{\circ} 51^{\prime} \mathrm{W}, 11$ August 1958, D.B.O. Savile 3785 (DAO 886692); Cunningham Inlet, $0.16 \mathrm{~km}$ from inlet, uplands, polar desert, $74^{\circ} 06^{\prime} \mathrm{N}, 93^{\circ} 55^{\prime} \mathrm{W}, 20 \mathrm{~m}$, 23 July 1976, L.C. Bliss s.n. (ALTA 56732); Cunningham Inlet, $8 \mathrm{~km}$ from inlet, uplands, polar desert, Som- erset plateau, $74^{\circ} 06^{\prime} \mathrm{N}, 93^{\circ} 55^{\prime} \mathrm{W}, 200 \mathrm{~m}, 20$ July 1976 , L.C. Bliss s.n. (ALTA 56731); west ridge overlooking Gull Canyon, $4 \mathrm{~km}$ east of Arctic Watch Lodge, dry rocky scree, with Xanthoria elegans, Festuca sp., $74^{\circ} 3^{\prime} 42.4^{\prime \prime} \mathrm{N}, 93^{\circ} 40^{\prime} 47.8^{\prime \prime} \mathrm{W}, 50 \mathrm{~m}, 7$ July 2013, $P$. Sokoloff 127 (CAN 603392); wet snowmelt valley at base of large unnamed mountain northwest of Arctic Watch Lodge, dry mud and clay mound in wet turfy tundra, with Festuca sp., 74²'31.3"N, 9351'1.7"W, 122 m, 9 July 2013, P. Sokoloff 181 (CAN 603393, NFM).

Saxifraga flagellaris ssp. platysepala (Trautvetter) A.E. Porsild (Spider Saxifrage, saxifrage à sépales larges, Kakillarnaliit) [circumpolar] - NUNAVUT: Somerset Island, mossy wet area outside whale biologist's cabin, $1.5 \mathrm{~km}$ north of Arctic Watch Lodge, wet mossy rocks, with Saxifraga cernua, Luzula confusa, Draba sp., Salix arctica, $74^{\circ} 4^{\prime} 58.8^{\prime \prime N}, 93^{\circ} 50^{\prime} 2^{\prime \prime W}, 94$ m, 8 July 2013, P. Sokoloff 156 (CAN 603394).

\section{Discussion}

Although five botanical collecting trips have taken place on Cunningham Inlet, the four trips previous to this study were focused primarily on selective collecting or ecological assessment. Combining these data with the 93 vascular plant specimens collected in 2013 provides a relatively complete inventory of the vascular plants of Cunningham Inlet.

Although the 48 vascular plant species documented is a relatively low number in terms of the species diversity over the entire island $(40 \%$ of those reported in Aiken et al. 2007, 48\% of those in Savile 1959, and $64 \%$ of those in Woo and Zoltai 1959), it is important to consider that Cunningham Inlet is well within the "cryptogam-herb barren" vegetation unit described in the Circumpolar Arctic Vegetation Map, which covers only roughly half of the island (Walker et al. 2005). The dominant growth forms described for this vegetation unit include "Cushion forbs: Papaver dahlianum ssp. polare; Draba; Potentilla hyparctica; Saxifraga oppositifolia" and "Graminoid: Alopecurus alpinus; Deschampsia borealis/brevifolia; Poa abbreviata; Puccinellia angustata; Phippsia; Luzula nivalis; Luzula confusa" (CAVM Team 2003*), nearly all of which are documented in this paper. Cunningham Inlet is also classified by the Circumpolar Arctic Vegetation Map Team as belonging to Arctic Bioclimate Subzone B, where prostrate dwarf shrubs (such as Arctic Willow) are the dominant growth form, and the number of species is estimated to run from 50 to 100 depending on the site (CAVM Team 2003*). Considering the ecology of Cunningham Inlet's polar desert and the five collecting trips to this site, it seems highly likely that we have documented all the vascular plants at this inlet and that the remaining species known for Somerset Island occur within the other ecosystems found on the island. 


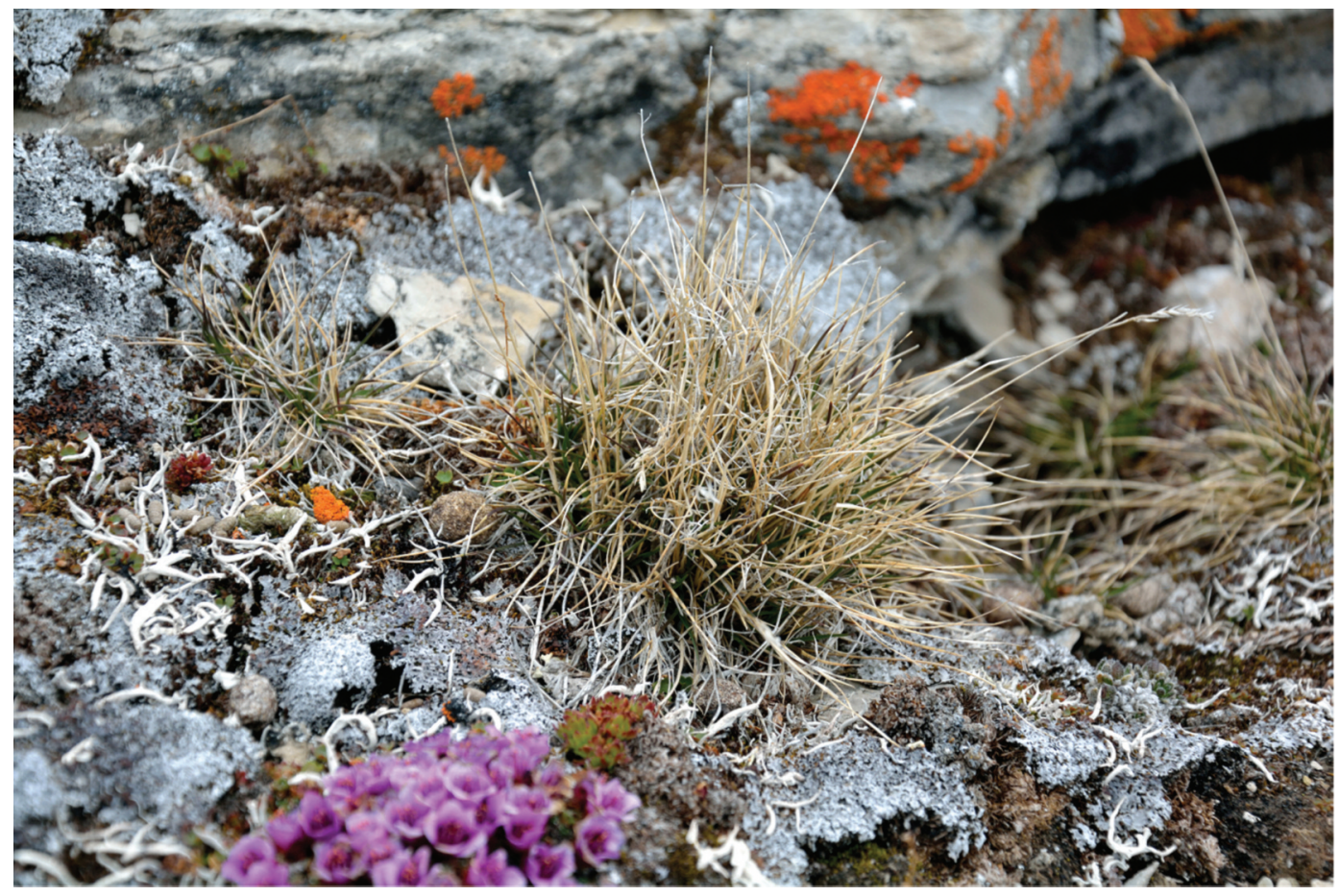

Figure 3: Edlund's Fescue (Festuca edlundiae S.G. Aiken, Consaul \& Lefkovitch) in habitat at the top of cliffs overlooking Gull Canyon, Somerset Island, Nunavut (Sokoloff 128). Photo by P. Sokoloff.

The summer of 2013 was unusually cold and late in the Canadian high Arctic (NASA 2013*), which delayed the flowering time of many species we encountered. Many species found on the open tundra had just begun their yearly growth, and the Purple Mountain Saxifrage, a benchmark spring ephemeral species in the Arctic, was still in full bloom when I left Cunningham Inlet. Although this resulted in taxonomically useful plant specimens rarely collected in flower (i.e., Saxifraga oppositifolia and Salix arctica), care should be taken to look for late-flowering specimens and specimens with fruits on subsequent visits to Cunningham Inlet. Thus, although comparisons between past and current vascular plant communities at this site are impossible given the paucity of earlier collections, in the future Cunningham Inlet could be used to monitor floristic change in the high Arctic using this inventory as a baseline, while keeping an eye out for any additions to the flora that would have been missed because of their later flowering time.

Two vascular plant species reported here, Festuca edlundiae (Figure 3) and Draba glabella, have not been reported before for Somerset Island (Savile 1959; Aiken et al. 2007). Festuca edlundiae is a widespread Canadian high Arctic endemic, found throughout the northern part of the archipelago, including Cornwallis and Prince of Wales Islands, adjacent to Somerset Island (Aiken et al. 2007). This species has only recently been recognized as a distinct taxon within the Canadian high Arctic Festuca brachyphylla complex (Aiken et al. 1995). Dwarf plants with a heavily marcescent habit, Festuca edlundiae, were first differentiated from the phenotypically variable Festuca hyperborea Holmen ex Frederiksen (High Arctic Fescue) based on isozymes (Aiken et al. 1995). Hybridization and introgression between Festuca edlundiae and the other high Arctic Festuca species have been documented (Saarela et al. 2013), but taxonomic boundaries between the various species are well understood and various keys exist separating the species using consistent morphologic characters (Fjellheim et al. 2001; Guldahl et al. 2001). Using these keys, it may yet be found that Festuca edlundiae has been collected on Somerset Island before its recognition as a distinct taxon; nonetheless Sokoloff 128 is the first known report of this grass species on the island.

Draba glabella (Sokoloff 134) is a first collection for both Somerset Island and the central Canadian Arctic archipelago. This species is common and widespread within the archipelago, but although its distribution extends from Banks to Baffin Island (east to west) and from the mainland to Ellesmere Island (south to north), it is absent from the central Arctic islands, including Bathurst, Prince William, and Cornwallis (Aiken et al. 2007), and has been reported only once on the west coast of Devon Island by Polunin (1940; 


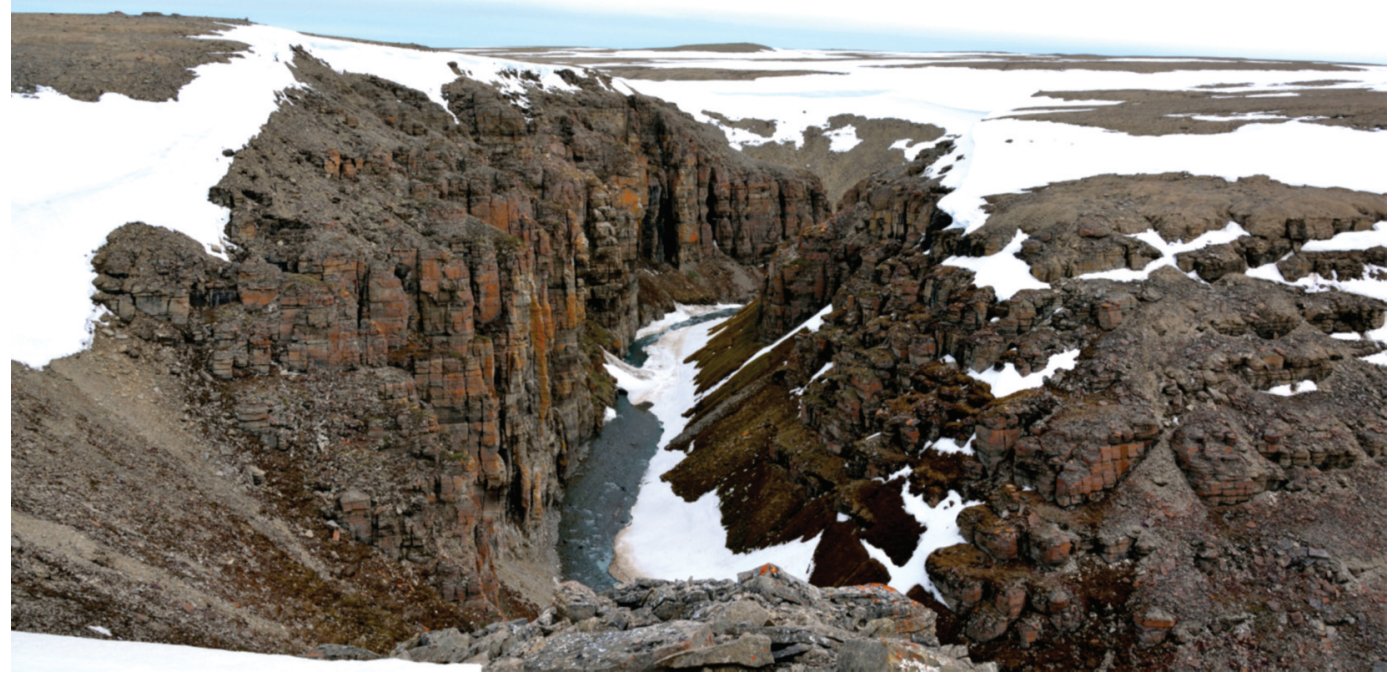

Figure 4: Habitat of Smooth Draba (Draba glabella Pursh) at Gull Canyon, Somerset Island, Nunavut. Draba glabella (Sokoloff 134) was collected at the base of the cliffs on the right side of the canyon. Photo by P. Sokoloff.

a second report in this volume is apparently a typo, a repetition of a collection made on Sugluk Island just off the coast of Quebec). These central islands are primarily polar desert (including the study site at Cunningham Inlet) and consist of shattered limestone with minimal tundra cover (Savile 1959; Bliss et al. 1984).

Accordingly, we found Draba glabella growing at only a single site: in wet moss and rich soil at a gull colony (Figure 4). Burt (2000) and Polunin (1940) indicate that such damp, nutrient- and soil-rich cliffs are ideal habitat for Draba glabella. Our discovery of this species at a bird colony in the middle of a gap in its range (Aiken et al. 2007) either points to bird-borne dispersal of the plant or indicates that this colony may serve as a refuge for this species in otherwise inhospitable and nutrient-poor habitat (Odasz 1994). In either case, other bird colonies within the central Canadian polar desert could harbour this widespread species as well.

The four identified species of marine algae, Fucus distichus, Alaria esculenta, Agarum cribrosum, and Sacharrina latissima, are all previously known to occur in the Barrow Straight, and both Alaria esculenta and Fucus distichus have been previously collected in Cunnigham Inlet (Lee 1980). Nostoc commune, known to be common in the Canadian high Arctic (Polunin 1947; Lennihan et al. 1994; Sheath et al. 1996), is poorly represented in Canadian algal collections (CANA, data available through Canadensys). Thus, Sokoloff 175 and 176 are likely the first known specimens of Nostoc commune from Somerset Island.

Although our 20 lichen and 13 bryophyte specimens greatly expand on known cryptogamic species from Somerset Island (Savile 1959), they were collected opportunistically and there are almost certainly gaps in the collection that a trained lichenologist or bryologist could fill.

\section{Acknowledgements}

I am immensely grateful to the following people who provided identification for many specialized taxonomic groups: Dr. Jeffery Saarela (Poacaeae, Cyperaceae, Juncaceae), Paul Hamilton (algae), Colin Freebury (lichens), Jennifer Doubt (bryophytes), Dr. Lynn Gillespie (Papaver, Potentilla), Étienne LéveilléBourret (Potentilla), and Gerry Mulligan (Draba). I thank Micheline Beaulieu-Bouchard, Jennifer Doubt, Amaina Boloron, Laura Smyk, and the Canadian Museum of Nature's botany volunteers for processing this collection. I thank Dr. Jeff Saarela for thoughtful comments on this manuscript. I also thank Gisele Mitrow and Amanda Ward for providing helpful access to the Agriculture and Agri-Food Canada collection of vascular plants. Comments by Dr. Jacques Cayouette and 
Dr. Paul Catling greatly enhanced earlier drafts of this work. This fieldwork was carried out as part of Arctic Watch Lodge's 2013 Steve Amarualik Youth Leadership Expedition and would not have been possible without the generous support of Arctic Watch Lodge and the Canadian Museum of Nature. In particular, I thank Richard Weber, Josée Auclair, Tessum Weber, Nansen Weber, and Ewan Affleck for organizing an exceptional expedition for the youth participants, for hosting me at Arctic Watch Lodge, and for going out of their way to contribute to the success of the collecting portion of the trip. Zach Halem and Alicia Malik both contributed as able collectors, and I commend all the expedition's youth - Alicia, Anika, Belinda, Louis, Savannah, Simeonie, Ryan, Sky, and Zach — for their enthusiasm, curiosity, and assistance. This research was conducted under Nunavut Department of Environment Wildlife research permit WL 2013-026.

Documents Cited (marked * in text)

Brouillet, L., F. Coursol, S. J. Meades, M. Favreau, M. Anions, P. Bélisle, and P. Desmet. 2010+. VASCAN, the database of vascular plants of Canada. Accessed 18 February 2014. http://data.canadensys.net/vascan/

CAVM (Circumpolar Arctic Vegetation Map) Team. 2003. Circumpolar Arctic vegetation map (1:7,500,000 scale). Conservation of Arctic Flora and Fauna map no. 1. United States Fish and Wildlife Service, Anchorage, Alaska, USA. ISBN: 0-9767525-0-6, ISBN-13: 978-0-9767525-0-9.

Elven, R., D. F. Murray, V. Y. Razzhivin, and B. A. Yurtsev. 2011. Annotated checklist of the panarctic flora (PAF): vascular plants. Panarctic Flora Project Steering Committee. Accessed 30 May 2014. http://www.nhm.uio.no/english /research/infrastructure/paf/

NASA (National Aeronautics and Space Administration). 2013. Arctic sea ice minimum in 2013 is sixth lowest on record. National Aeronautics and Space Administration, Washington, D.C., USA. Accessed 14 March 2014. http: //www.nasa.gov/content/goddard/arctic-sea-ice-minimumin-2013-is-sixth-lowest-on-record/\#.UyNMTj9dV8E

Woo, V. B., and S. Zoltai. 1977. Reconnaissance of the soils and vegetation of Somerset and Prince of Wales Islands, N.W.T. Information Report NOR-X-186. Northern Forest Research Centre, Edmonton, Alberta, Canada. 127 pages.

\section{Literature Cited}

Aiken, S. G., L. L. Consaul, and L. P. Lefkovitch. 1995. Festuca edlundiae (Poaceae), a high arctic, new species compared enzymatically and morphologically with similar Festuca species. Systematic Botany 20: 374-392.

Aiken, S. G., M. J. Dallwitz, L. L. Consaul, C. L. McJannet, R. L. Boles, G. W. Argus, J. M. Gillett, P. J. Scott, R. Elven, M. C. LeBlanc, L. J. Gillespie, A. K. Brysting, H. Solstad, and J. G. Harris, 2007. Flora of the Canadian Arctic Archipelago. NRC Research Press, National Research Council of Canada, Ottawa, Ontario, Canada. Accessed 18 February 2014. http://nature.ca/aaflora/data.
Bliss, L. C., J. Svoboda, and D. I. Bliss. 1984. Polar deserts, their plant cover and plant production in the Canadian high Arctic. Holarctic Ecology 7: 305-324.

Burt, P. 2000. Barrenland Beauties: Showy Plants of the Canadian Arctic. Outcrop Ltd. Yellowknife, Northwest Territories, Canada. 238 pages.

Fjellheim, S., R. Elven, and C. Brochmann. 2001. Molecules and morphology in concert. II. The Festuca brachyphylla complex (Poaceae) in Svalbard. American Journal of Botany 88: 869-882.

Guldahl, A. S., L. Borgen, and I. Nordal. 2001. Variation in the Festuca brachyphylla (Poaceae) complex in Svalbard, elucidated by chromosome numbers and isozymes. Botanical Journal of the Linnean Society 137: 107-126.

Lee, R. K. S. 1980. A Catalogue of the Marine Algae of the Canadian Arctic. Publication in Botany 9. National Museums of Canada, Ottawa, Ontario, Canada. 82 pages.

Lennihan, R., D. M. Chapin, and L. G. Dickson. 1994. Nitrogen fixation and photosynthesis in high arctic forms of Nostoc commune. Canadian Journal of Botany 72: 940945.

Mallory, C., and S. Aiken. 2012. Common plants of Nunavut. Inhabit Media Inc. Iqaluit, Nunavut, Canada. 205 pages.

Odasz, A. 1994. Nitrate reductase activity in vegetation below an arctic bird cliff, Svalbard, Norway. Journal of Vegetation Science 5: 913-920.

Polunin, N. 1940. Botany of the Canadian Eastern Arctic. Part I: Pteridophyta and Spermatophyta. National Museum of Canada Bulletin 92. National Museum of Natural Sciences, Ottawa, Ontario, Canada. 408 pages.

Polunin, N. 1947. Botany of the Canadian Eastern Arctic. Part II: Thallophyta and Bryophyta. National Museum of Canada Bulletin 97. National Museum of Natural Sciences, Ottawa, Ontario, Canada. 573 pages.

Porsild, A. E., and W. J. Cody. 1980. Vascular Plants of the Continental Northwest Territories, Canada. National Museum of Natural Sciences, Ottawa, Ontario, Canada. 667 pages.

Saarela, J. M., P. C. Sokoloff, L. J. Gillespie, L. L. Consaul, and R. D. Bull. 2013. DNA barcoding the Canadian Arctic flora: core plastid barcodes (rbcL + matK) for 490 vascular plant species. PLoS One 8: e77982.

Savile, D. B. O. 1959. The botany of Somerset Island, District of Franklin. Canadian Journal of Botany 37: 959-1002.

Sheath, R. G., M. L. Vis, J. A. Hambrook, and K. M. Cole. 1996. 7. Tundra stream macroalgae of North America: composition, distribution and physiological adaptations. Hydrobiologia 336: 67-82.

Smith, T. G., and B. Sjare. 1990. Predation of belugas and narwhals by polar bears in nearshore areas of the Canadian High Arctic. Arctic 43: 99-102.

Smith, T. G., and A. R. Martin. 1994. Distribution and movements of belugas, Delphinapterus leucas, in the Canadian High Arctic. Canadian Journal of Fisheries and Aquatic Sciences 51: 1653-1663.

Walker, D. A., M. K. Raynolds, F. J. A. Daniëls, E. Einarsson, A. Elvebakk, W. A. Gould, and A. E. Katenin. 2005. The Circumpolar Arctic vegetation map. Journal of Vegetation Science 16: 267-282.

Received 25 March 2014

Accepted 17 June 2014 